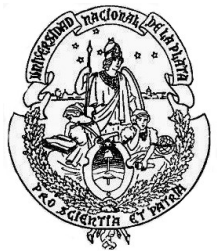

Maestría en Economía

Facultad de Ciencias Económicas

Universidad Nacional de La Plata

Tesis de Maestría

TíTULO

Efectos de la desregulación del sistema universitario en el mercado laboral en Perú

\author{
Alumno \\ César Edinho Del Pozo Loayza \\ DirectorA \\ María LaUra Alzúa
}

INFORME FINAL

Agosto 2018 


\title{
Efectos de la desregulación del sistema universitario en el mercado laboral en Perú \\ CÉsar Del Pozo LoAyza ${ }^{1}$
}

\begin{abstract}
In 1995 the peruvian's university system was reformed, the requirements for the entry of new universities were relaxed, the result of this reform was an relevant increases in the number of universities (around 218\% between 1996 and 2017). The reform caused a significant proportion of workers, especially those born after 1980 to pursue higher education, which in the absence of the reform would they not have been able to access to the university system. Differences in the exposure to the reform in terms of the workers' year of birth, allow me to recover positive causal effects of higher education on wages and labor conditions, mainly a lower probability of labor informality.
\end{abstract}

JEL Codes: I23, I26, J31, J81,C6

Keywords: Higher education, instrumental variables, wage premium, labor informality

\section{Resumen}

En 1995 se reformó el sistema universitario peruano, flexibilizando los requerimientos para la entrada de nuevas universidades. El resultado de esta reforma fue un aumento relevante en el número de universidades (alrededor del $218 \%$ entre 1996 y 2017). La reforma generó que una proporción significativa de trabajadores, especialmente aquellos nacidos después de 1980, alcancen estudios superiores, que en ausencia de ella, no habrían podido acceder al sistema universitario. Diferencias en la exposición a la reforma, en términos del año de nacimiento de los trabajadores, permiten recuperar los efectos causales positivos de la educación superior sobre los salarios y mejoras las condiciones laborales, principalmente una menor probabilidad de informalidad laboral.

Códidos JEL: I23, I26, J31, J81,C6

Palabras clave:Educación universitaria, resultados laborales, variables instrumentales, premio salarial, informalidad laboral

\footnotetext{
${ }^{1} \mathrm{El}$ presente documento constituye el informe final de Tesis de Maestría en Economía del Departamento de Economía de la FCE-UNLP. Este estudio fue financiado por la Unidad de Becas de Brot fuer die Welt (Alemania) y el Programa de apoyo a tesis de maestría del ARES (Bélgica). El autor agradece los comentarios y sugerencias de Joaquín Serrano y los participantes del seminario de avances de tesis de maestría del Departamento de Economía de la FCE-UNLP.
} 


\section{Índice}

1. Introducción 3

2. Contexto 4

2.1. Desregulación del sistema universitario en Perú . . . . . . . . . . . 4

2.2. Revisión de la literatura . . . . . . . . . . . . . . . . . . . . . 7

3. Metodología 9

3.1. Fuentes de datos . . . . . . . . . . . . . . . . . . . . . . . 9 9

3.2. Estrategia de identificación . . . . . . . . . . . . . . . . . . . 9

3.3. Estrategia empírica . . . . . . . . . . . . . . . . . . . . . 12

4. Resultados 16

4.1. Descripción de los datos . . . . . . . . . . . . . . . . . . . . . . . 16

4.2. Efectos de la desregulación en resultados laborales . . . . . . . . . . . . 17

4.2.1. Resultados I: Modelo de forma reducida . . . . . . . . . . . . 17

4.2.2. Resultados II: Modelo de Variables Instrumentales . . . . . . . . 20

4.2.3. Resultados III: Heterogeneidades . . . . . . . . . . . . . . . . . 24

4.3. Pruebas de robustez: experimentos falsos . . . . . . . . . . . . . . . . . 28

5. Conclusiones 31

$\begin{array}{ll}\text { Apéndices } & 35\end{array}$ 


\section{Introducción}

En la década de los noventa muchos países de Latinoamérica expandieron la cobertura de sus sistemas universitarios, en promedio en la región la tasa de matricula universitaria pasó de $17 \%$ en 1991 a $43 \%$ en 2012 [Espinoza y Urzúa 2015]. En tal contexto, en Perú desde 1995 se implementó una reforma de política que flexibilizó los requerimientos para la entrada de nuevas universidades e incentivó la inversión privada en el sistema universitario. Esta reforma implicó una significativa desregulación del mercado de educación superior, que motivó un importante crecimiento en la oferta de universidades, el número de universidades privadas se incrementó en $218 \%$ entre 1996 y 2017.

A modo de contextualizar la reforma universitaria descrita: Brasil con una población de más de 200 millones de habitantes cuenta con aproximadamente 197 universidades; mientras que Perú, con una población de alrededor de 32 millones tiene 140 universidades. Por ello, Perú se constituye como el segundo país con más universidades en sudamérica, actualmente alrededor del $25 \%$ de estudiantes universitarios peruanos pertenecen a universidades creadas a partir de la reforma de 1995.

La expansión de la oferta de universidades afectó las decisiones de educación superior de determinado grupo de individuos, particularmente aquellos que nacieron después de 1980, para los cuales los requisitos de admisión al sistema universitario fueron más flexibles. Esto permitió que la proporción de trabajadores con estudios universitarios se incremente considerablemente en el mercado laboral. Lo anterior sugiere la siguiente cuestión: ¿Cuáles fueron los efectos de la desregulación del sistema universitario peruano en el acceso a la educación superior y en los resultados del mercado laboral?

Para tratar de responder esa pregunta, en este estudio se ha empleado un diseño de evaluación de impacto no experimental basado en variables instrumentales, en la cual se han identificado individuos más y menos expuestos al proceso de desregulación del sistema universitario en función a su año de nacimiento, los individuos más expuestos serían aquellos nacidos luego de 1980 y los menos expuestos aquellos nacidos antes de dicho año. La desregulación permitió que individuos con estudios secundarios accedan a la educación superior que de otro modo no lo habrían logrado. Para recuperar efectos causales de la educación en resultados laborales, la aproximación por variables instrumentales, ha sido ampliamente utiliza en la literatura [Card 1999] y en particular para evaluar el efecto de la educación universitaria en el mercado laboral, entre otros, por: [Lemieux y Card 2001] y [Maurin y McNally 2008].

En este estudio se ha planteado una estrategia de identificación que permitió estimar de forma plausible los potenciales efectos de la desregulación del sistema universitario en algunos resultados laborales de interés. En particular, se han identificado efectos 
causales de la educación universitaria en los salarios y la informalidad laboral. Se encuentra que por cada año de estudios superiores en universidades creadas luego de la reforma, el premio salarial es de 13.5 puntos porcentuales; mientras que, por cada año de estudios universitarios la probabilidad de empleo informal (probabilidad de no tener seguro de salud ni afiliación al sistema de pensiones jubilatorias) se reduce en 11.3 puntos porcentuales.

El resto del documento se organiza de la siguiente manera: en la sección 2 se describe el proceso de desregulación del sistema universitario peruano y se realiza una breve revisión de la literatura relacionada con los objetivos de investigación. En la sección 3 se detalla la fuente de datos empleados, se plantea la estrategia de identificación y se describe la estrategia econométrica. En la sección 4 se describen y discuten los principales resultados empíricos y finalmente en la sección 5 se mencionan algunas conclusiones.

\section{Contexto}

\subsection{Desregulación del sistema universitario en Perú}

En Perú a partir de 1995 se modificó la estructura legal que regulaba la entrada al mercado de nuevas universidades. En dicho año, el gobierno peruano creó el Concejo Nacional para la Autorización del Funcionamiento de Universidades (CONAFU), este organismo estatal fue el encargado de evaluar y emitir autorizaciones para el funcionamiento de nuevas universidades a nivel nacional. Previamente a dicha modificación, las universidades sólo podían ser creadas por ley del Congreso de la República [Lavado et al. 2014]. Adicionalmente, se promulgó la Ley para Promover la Inversión en Educación, con ella se autorizó que los excedentes que podrían surgir al final del ejercicio presupuestario de las universidades podrían ser distribuidos entre sus inversionistas, anteriormente a la reforma tales excedentes sólo podían ser invertidos en nombre de las universidades o destinado a becas de estudios. Esta normativa adicional motivó aún mas el incremento de universidades principalmente privadas lo cual se hizo evidente a partir del año 2010.

El resultado de tales cambios normativos para la creación y gestión de universidades, entendido en este estudio como la desregulación del sistema universitario peruano, fue el notable incremento del número de universidades, principalmente, universidades privadas. Como se muestra en la Figura 1, al año 1995 en el Perú operaban 56 universidades, actualmente operan 140 (89 privadas y 51 públicas). El número de universidades privadas y públicas se incrementó en $218 \%$ y $82 \%$, respectivamente. Solamente entre 2009 y 2011 se crearon 40 universidades nuevas. 
Figura 1: Perú: Número de universidades 1990-2017

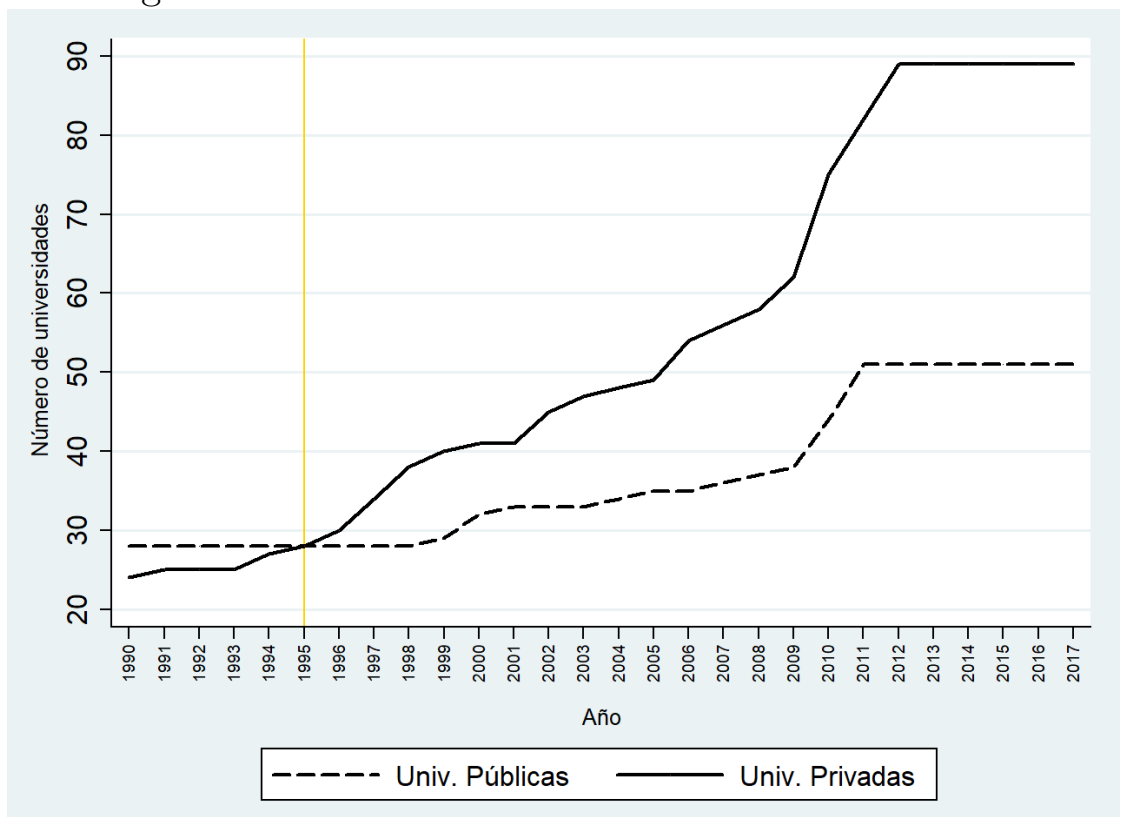

Fuente: Superintendencia Nacional de Educación Superior (SUNEDU).

Luego de la desregulación, el sistema universitario presenta las siguientes características $^{2}$ : (i) Las nuevas universidades muestran una menor selectividad en el proceso de admisión, éstas admiten en promedio al $91 \%$ de sus postulantes. (ii) Las nuevas universidades (privadas) muestran menores costos de matrícula y enseñanza respecto de universidades pre-establecidas en el sistema, el costo anual en este tipo de universidades es, en promedio, $32 \%$ menos que en universidades establecidas (universidades creadas antes de 1995). (iii) Se generó un shock en la oferta de universidades menos selectivas, a finales de 2013, se registraron más de un millón de alumnos universitarios, de los cuales $25 \%$ pertenecen a las nuevas universidades entrantes. (iv) Respecto de algunos indicadores de calidad educativa (ratio graduados/matriculados, titulados/matriculados) las nuevas universidades muestran en general peores resultados. En particular, [Lavado et al. 2014], [Lavado et al. 2015] y [Yamada et al. 2015], sugieren que las universidades entrantes ofrecerían en general una menor calidad educativa respecto de universidades establecidas.

Con información de trabajadores asalariados y ocupados entre 24 y 65 años en conglomerados urbanos de la Encuesta Nacional de Hogares (Enaho) entre 2014 y 2016, en la Figura 2 (a) se observa que el premio salarial en el mercado laboral es diferente en función al nivel educativo alcanzado por los individuos. En promedio, los individuos que egresaron universidades entrantes obtienen salarios horarios 1.6 veces mayores que individuos con nivel secundario (USD 3.2 y USD 2, respectivamente).

Adicionalmente, la desregulación del sistema universitario pudo tener efectos en las

\footnotetext{
${ }^{2}$ Más detalles en el Cuadro 8 del Apéndice A.
} 
Figura 2: Resultados laborales por nivel educativo alcanzado (Individuos entre 24 y 65 años de edad)

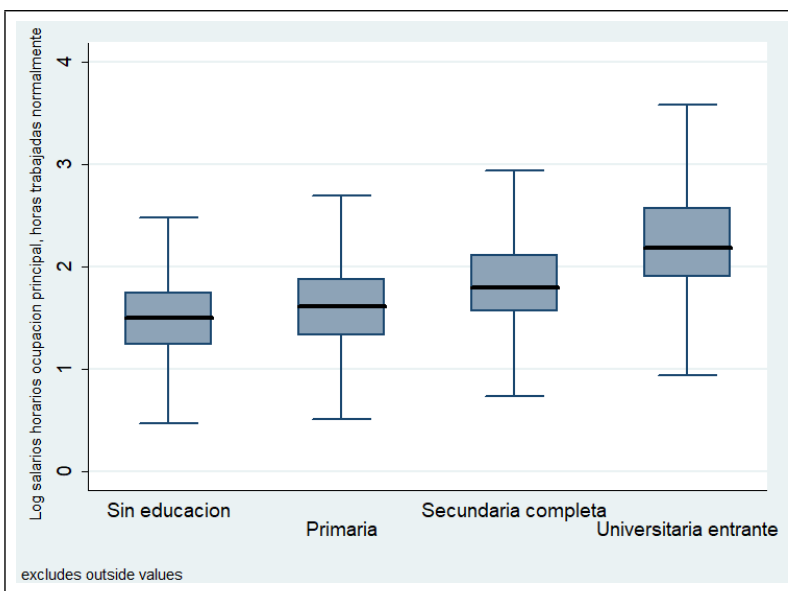

(a) (Log) Salarios horarios

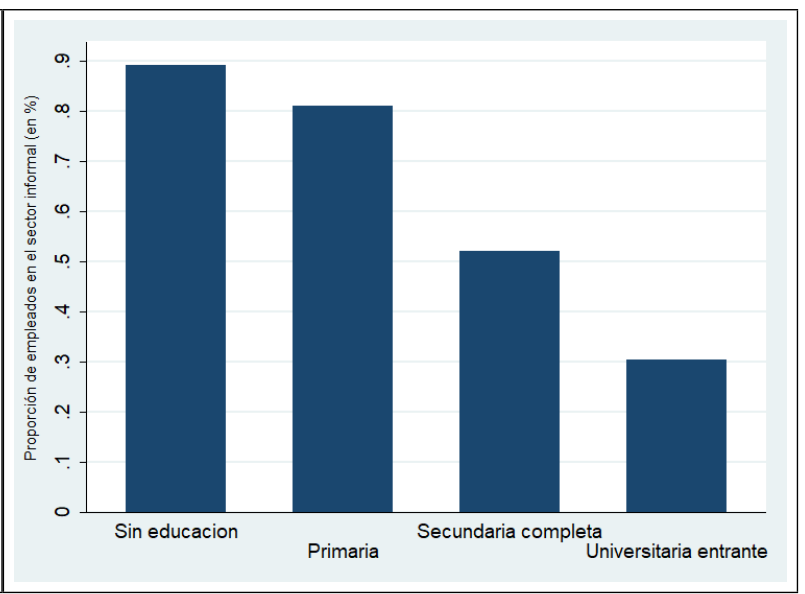

(b) Probabilidad de trabajar en el sector informal Nota: Individuos ocupados y asalariados en actividad principal (áreas urbanas). Fuente: Encuesta Nacional de Hogares 2014-2016. Elaboración Propia.

condiciones laborales que obtienen los egresados en el mercado laboral, es decir, en la probabilidad de estar empleados en el sector informal ${ }^{3}$. En la Figura 2 (b), se observa que la proporción de trabajadores informales con educación secundaria completa es $53 \%$; mientras que, dicha proporción en trabajadores con educación superior egresados de universidades entrantes es $30 \%$.

En la figura anterior se observan algunos hechos estilizados congruentes con la literatura de economía laboral [Borjas 2013]: (i) Los trabajadores más educados obtienen un premio salarial y mejores condiciones laborales por su mayor cualificación, lo cual sugeriría que la educación incrementaría la productividad laboral o en su defecto serviría como una señal de mayor habilidad ante los empleadores. (ii) Los resultados laborales mejoran con la calificación (más salarios y menor informalidad) a una tasa relativamente constante para individuos menos calificados y a una tasa creciente para individuos con estudios universitarios.

\footnotetext{
${ }^{3}$ De acuerdo con el Instituto Nacional de Estadística e Informática (INEI), se consideran trabajadores informales asalariados aquellos individuos que no cuentan con ningún seguro de salud, ni cobertura de pensiones.
} 


\subsection{Revisión de la literatura}

\section{Literatura relacionada sobre el efecto de la educación en resultados laborales}

Considerando que la educación juega un papel importante en los resultados laborales, la literatura empírica ha explorado ampliamente la correlación positiva entre educación y salarios [Card 1999]. Usualmente, esta relación a sido aproximada empíricamente a través de la denominada Ecuación de Mincer [Mincer 1974]; no obstante, en ausencia de evidencia experimental, resulta difícil establecer relaciones causales entre mayor niveles educativos y los resultados laborales de interés. Entre los principales problemas empíricos para la interpretación causal de la Ecuación de Mincer se tiene la presencia de variables omitidas no observables no incluidas en la ecuación de salarios (principalmente la habilidad) y la presencia de errores de medición tanto en la variable de salarios como en la variable de educación.

Para abordar explícitamente los problemas empíricos en la Ecuación de Mincer, en la literatura se han empleado, principalmente, aproximaciones a través de Variables Instrumentales (IV, por sus siglas en inglés). En dicha aproximación un instrumento válido es alguna variable que no tiene asociación directa con los los resultados laborales sino solamente a través de su efecto en la educación. En la literatura, los instrumentos provienen principalmente de tres fuentes [Card 1999]: (i) Instrumentos basados en características familiares de los individuos como proxies de habilidad ([Ashenfelter y Zimmerman 1997] y [Ashenfelter y Rouse 1998]); (ii) Instrumentos basados en muestras de hermanos gemelos o mellizos, en los cuales se asume que la habilidad inobservable es similar ([Ashenfelter y Rouse 1998] y [Behrman et al. 1994]); (iii) Instrumentos basados en el conocimiento institucional de los sistemas educativos y la ocurrencia de cambios en los sistemas educativos generados por algún tipo de reforma política ( [Angrist y Krueger 1991], [Card 1995], [Duflo 2001], [Lemieux y Card 2001] y [Maurin y McNally 2008]).

En particular, los cambios en los sistemas educativos denominados como "experimentos naturales" o "experimentos de política", permiten una fuente de variación exógena que servirían para recuperar efectos causales entre la educación y los resultados laborales. En el trabajo empírico, este tipo de experimento permitiría comparar trabajadores con el mismo nivel esperado de habilidad no observable ([Borjas 2013]).

Para los objetivos del presente estudio, dentro de la literatura relacionada con el efecto de la educación universitaria en los ingresos laborales se tienen los siguientes estudios: en primer término, [Lemieux y Card 2001] evalúan el efecto en los salarios del notable incremento en la matrícula universitaria en Canadá, generada por un programa de beneficios para veteranos de la Segunda Guerra Mundial (VRA, por sus siglas en inglés) para promover su inserción al mercado laboral a través de incrementar la 
matriculación y asistencia a universidades. El programa tuvo una mayor intensidad en la provincia de Ontario en individuos cuya edad se encontrada entre los 18 y 21 años en 1945, los autores emplean como grupo de control a los individuos residentes en la provincia de Quebec donde dicho programa prácticamente no tuvo efectos. Los autores emplean la interacción entre la cohorte de nacimiento (entre 18 y 21 años en 1945) y la provincia de nacimiento (Ontario o Quebec) como un instrumento de los años de educación, encuentran que el programa habría generado elevados retornos marginales a la educación universitaria; en particular, estiman un premio salarial de 15 puntos porcentuales.

Por su parte, [Maurin y McNally 2008] emplean la denominada revolución de 1968 como un experimento de política para estimar el efecto de la educación superior en los salarios para el caso de Francia. En 1968, el sistema universitario flexibilizó los requerimientos de admisión a las universidades, beneficiando a una determinada cohorte de individuos; en particular, aquellos nacidos entre los años 1947 y 1949. Los autores emplean dummies por año de nacimiento como instrumentos de la educación superior y encuentran que por cada año adicional de estudios universitarios los salarios de los individuos beneficiados se incrementaron en 14 puntos porcentuales, los autores argumentan que dicho premio salarial capturaría el efecto de la educación en el capital humano de los trabajadores.

\section{Literatura relacionada para el caso peruano}

Los efectos del proceso de desregulación del sistema universitario en el mercado laboral es una problemática que es estudiada recientemente. Para el caso peruano los estudios disponibles ha empleado básicamente ecuaciones de Mincer y estimado los coeficientes de interés por OLS sin dar cuenta de los potenciales problemas de endogenidad en dicho estimador. Entre ellos: [Psacharopoulos 1994] estima retornos de 10 puntos porcentuales; mientras que, [Espinoza y Urzúa 2015], estiman que el retorno promedio de los estudios superiores en universidades entrantes sería de 24 puntos porcentuales.

Por su parte, Yamada et al., (2015) emplean información de la encuesta de hogares de 2014 y una ecuación de salarios, para abordar el problema de endogeneidad emplean como instrumento de la educación el año de creación de la universidad; no obstante, se considera que dicho instrumento podría estar correlacionado con el término de error en la ecuación de salarios, puesto que en un año de bonanza económica podrían crearse más universidades dada las mejores condiciones para la inversión. No obstante, los autores encuentran que acceder a estudios superiores en una universidad entrante implicaría un premio salarial de 71 puntos porcentuales. 


\section{Metodología}

\subsection{Fuentes de datos}

La evidencia empírica del estudio se basa principalmente en la Encuesta Nacional de Hogares (ENAHO) correspondiente a los años 2014, 2015 y 2016 en una estructura de datos de tipo pooled. Enaho es implementada anualmente por el Instituto Nacional de Estadística e Informática (INEI).

En dicha base de datos es posible identificar la universidad de estudios superiores de los individuos, el análisis se concentró en individuos entre 24 y 65 años que residen en áreas urbanas, ocupados y asalariados en actividades principales (no se incluyeron trabajadores cuentapropistas). Los individuos fueron agrupados en diversas cohortes en función al año de nacimiento. En ENAHO también está disponible información respecto de participación e ingreso laboral, condiciones laborales; es decir si los individuos trabajan en condiciones de informalidad y un amplio conjunto de características demográficas y socioeconómicas.

La muestra empleada en este estudio consta de información de 40 mil individuos, de los cuales 8 mil tienen estudios primarios, 11 mil alcanzaron nivel secundario, 9 mil estudios técnicos, 1.3 mil estudiaron en universidades entrantes y 11 en universidades establecidas. El efecto de la desregulación del sistema universitario peruano se ha identificado sobre una sub-muestra constituida por trabajadores con estudios secundarios y universitarios en universidades entrantes.

\subsection{Estrategia de identificación}

Para identificar de forma plausible los potenciales efectos de la desregulación del sistema universitario en los resultados laborales de interés, en el presente estudio se considera a dicho proceso como un "experimento de política", el cual habría afectado de manera heterogénea a los individuos en función a su año de nacimiento. Se asume que el año de nacimiento que determina un mayor o menor grado de exposición a la desregulación es una característica exógena a los resultados laborales, puesto que la habilidad no observable no estaría correlacionada con el año de nacimiento.

En la Figura 3, se muestra la estructura del mercado laboral peruano en términos de la proporción de trabajadores asalariados nacidos entre 1965 y 1990 por nivel educativo alcanzado. La mayor calificación de los trabajadores se concentra en el nivel secundario (41\%), seguido por universitario (28\%), nivel técnico o terciario $(21 \%)$ y en menor proporción nivel primario $(10 \%)$. 
Figura 3: Perú: Nivel educativo de la fuerza laboral por año de nacimiento

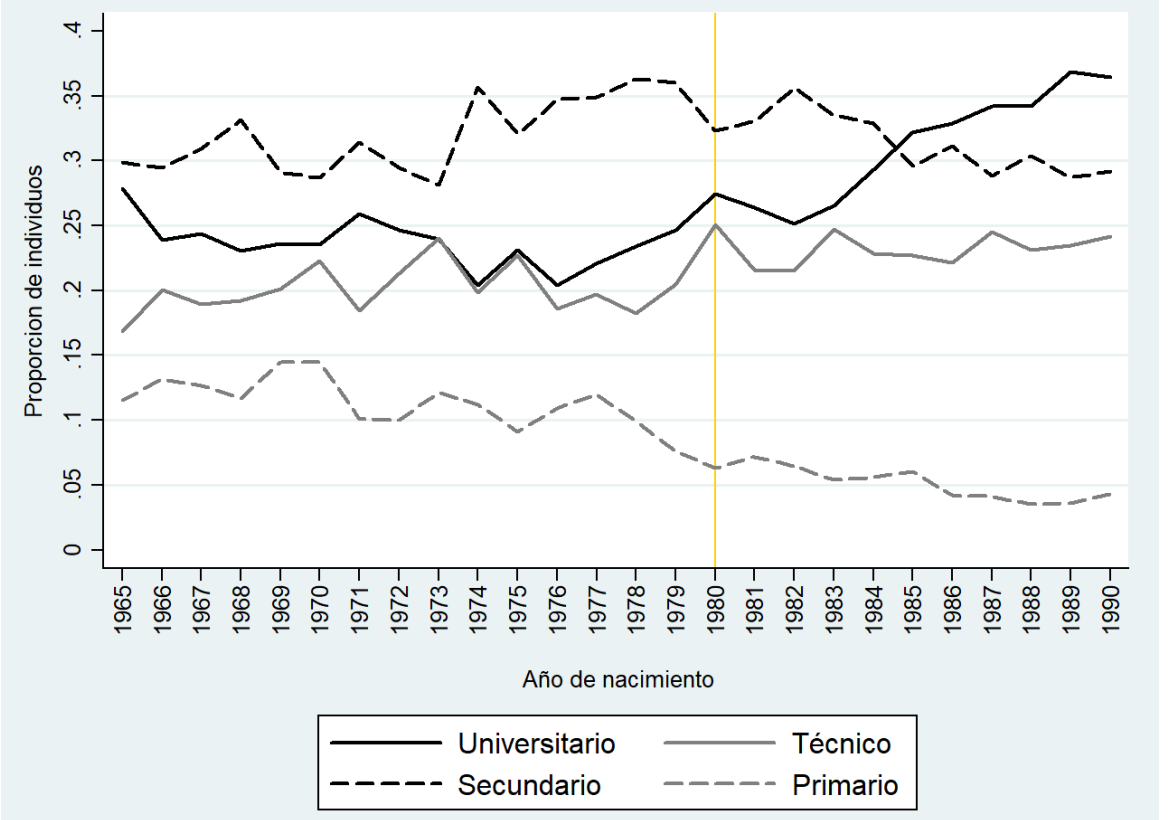

Nota: Individuos nacidos entre 1965 y 1990.

Fuente: Encuesta Nacional de Hogares 2014-2016.

Elaboración Propia.

Se observa que la proporción de trabajadores con estudios universitarios se incrementa a partir de individuos nacidos el año 1976, resultado congruente el incremento en la valoración de los estudios universitarios en diversos países de América Latina ([Espinoza y Urzúa 2015]). No obstante, dicha tendencia es aún más marcada a partir de la cohorte de individuos nacidos en 1980, la proporción de trabajadores con estudios universitarios pasó de $27 \%$ en dicha cohorte a $36 \%$ en la cohorte de 1990; en contraste, se observa una tendencia decreciente en la proporción de trabajadores con estudios secundarios entre similares cohortes.

Por su parte, la proporción de trabajadores con estudios técnicos se mantendría constante entre cohortes; mientras que, la proporción de trabajadores con estudios primarios se ha reducido considerablemente en el tiempo.

En la Figura 4, se muestra la proporción de individuos nacidos entre los años 1965 y 1990 por niveles educativos, los cuales habrían sido afectados por la desregulación del sistema universitario peruano: trabajadores con estudios secundarios completos y trabajadores con estudios universitarios en universidades entrantes (universidades creadas después de la desregulación de 1995). En general, se observa que a partir de la cohorte de nacidos en 1980 en adelante la proporción de trabajadores con estudios secundarios se reduce; mientras que, la proporción de trabajadores con estudios superiores en universidades entrantes crece considerablemente desde la cohorte de 1980 en adelante. 
Figura 4: Perú: Grupos expuestos a la desregulación universitaria por año de nacimiento

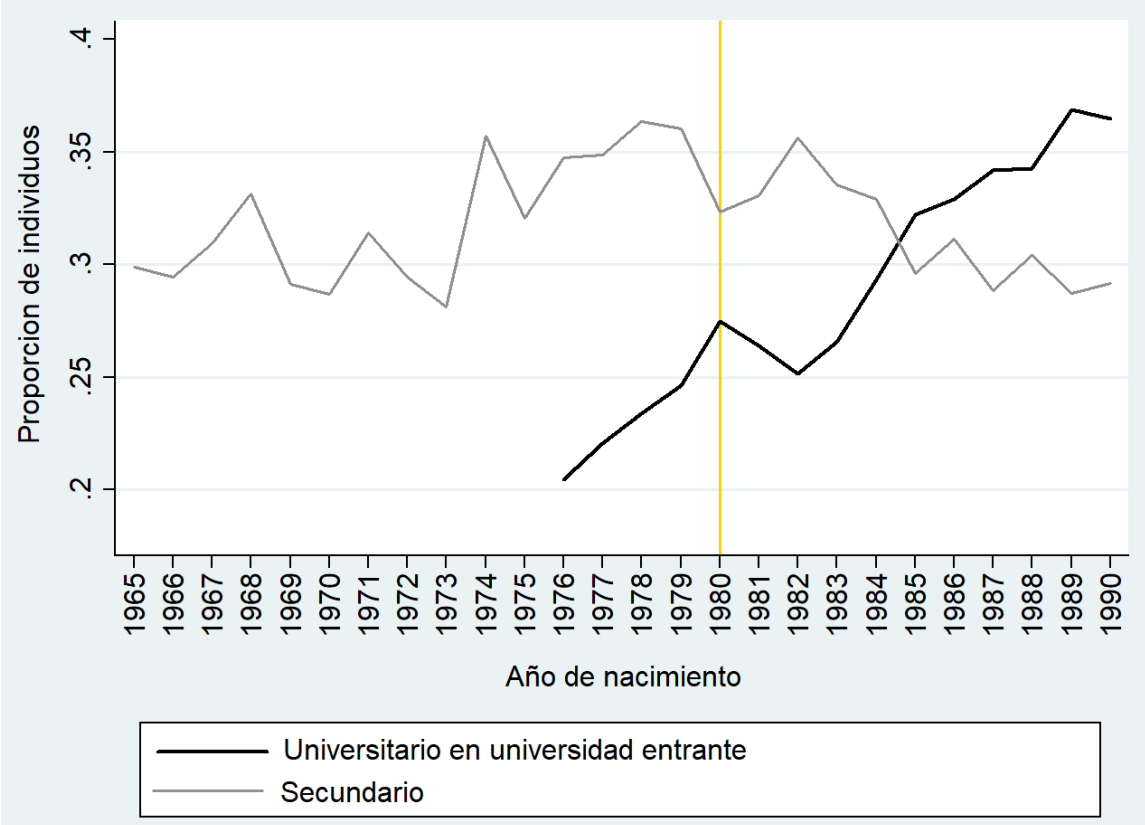

Nota: Individuos nacidos entre 1965 y 1990.

Fuente: Encuesta Nacional de Hogares 2014-2016.

Elaboración Propia.

Para el caso peruano, los individuos pueden iniciar estudios universitarios sólo si lograron concluir los estudios secundarios (en general a los 16 años), dado que la desregulación se inició a partir de 1995, los individuos nacidos luego del año 1980 estarían más expuestos al incremento en la oferta de universidades, principalmente universidades entrantes.

Para la estimación de los efectos de la desregulación en los resultados laborales se consideraron solamente individuos con secundaria completa e individuos que estudiaron en universidades entrantes, se asume que entre ambos grupos de comparación, en promedio, en nivel de características no observables (principalmente habilidad no observada) es similar. En otros términos, se asume que de no ser por la desregulación del sistema universitario, que posibilitó la entrada de universidades con menores criterios de selectividad de postulantes y flexibilizando la admisión ([Lavado et al. 2014]), aquellos individuos con secundaria completa no habrían tenido acceso a la educación universitaria. Este supuesto podría permitir estimar el premio laboral que capturaría el efecto "puro"de la educación universitaria en el capital humano ([Maurin y McNally 2008]).

De acuerdo con la Figura 4, se asume que la exposición a la desregulación del sistema universitario es una función creciente con el año de nacimiento desde 1980 en adelante, la cual habría incrementado el acceso a la educación superior en individuos con estudios secundarios, quiénes en ausencia de la desregulación no habrían podido acceder al sistema universitario peruano. 


\subsection{Estrategia empírica}

Para estimar los efectos de la desregulación en el mercado laboral se compararon los resultados laborales de dos cohortes de individuos: (i) Individuos nacidos a partir de 1980 (grupo de tratamiento); (ii) Individuos nacidos antes de 1980 (grupo de control). Esta comparación puede proveer una evaluación del efecto promedio en ingresos y condiciones laborales del acceso a la educación superior experimentada por el grupo de individuos mayormente expuestos a la desregulación del sistema universitario peruano.

De acuerdo con [Maurin y McNally 2008], se considera que los resultados laborales $\left(Y_{i}\right)$ del individuo i, que pertenece a la cohorte $\left(c_{i}\right)$ a la edad $\left(a_{i}\right)$, están determinados por:

$$
y_{i}=\alpha_{1}+\delta\left(s_{i}\right)+\gamma_{1}\left(c_{i}\right)+\gamma_{2}\left(a_{i}\right)+X_{i}^{\prime} \lambda_{1}+\mu_{i, j}+\varepsilon_{i}
$$

En la ecuación anterior, $Y_{i}$ es un vector de resultados laborales de interés: (i) (Logaritmo) Salario horario, calculado como el producto de los ratios ingresos anuales/semanas trabajadas y semanas trabajadas/horas trabajadas típicamente por semana ([Card 1999]); (ii) (Logaritmo) del los ingresos anuales; (iii) Variable binaria igual a 1 si el individuo trabaja en un empleo informal y 0 de otro modo; (iv) Variable binaria igual a 1 si el individuo no cuenta con contrato de trabajo y 0 de otro modo; (v) Variable binaria igual a 1 si el individuo no cuenta con afiliación al sistema de pensiones jubilatorias.

Por su parte, $s_{i}$ es una variable binaria que es igual a 1 si el individuo estudió en una universidad entrante y 0 si cuenta con secundaria completa, se considera además a esta variable como una continua en términos de años de educación universitaria ([Park 1996] y [Lemieux y Card 2001]). $X_{i}^{\prime}$ es un vector de características de control (sexo y raza). Se consideran además $\mu_{i, j}$ efectos fijos a nivel de la j-región de residencia. Finalmente, $\varepsilon_{i}$ representa determinantes inobservables de los resultados laborales.

Considerando la edad constante, la tendencia de la cohorte de nacimiento es equivalente a un tendencia temporal que captura el efecto de los cambios en la productividad en los resultados laborales en el tiempo. Asumiendo que la educación universitaria $\left(s_{i}\right)$ varía de forma no lineal entre cohortes, esta variable puede ser expresada por:

$$
s_{i}=\alpha_{2}+\sum_{t=1980}^{1990} \eta_{t} d_{i, t}+\theta\left(c_{i}\right)+X_{i}^{\prime} \lambda_{2}+\mu_{i, j}+\nu_{i}
$$

En la ecuación anterior, $d_{i, t}$ son variables dummies que son iguales a 1 para cada año o cohorte de nacimiento entre los años 1980 y 1990 (la categoría de referencia son individuos pertenecientes a las cohortes entre 1965 y 1979), los cuales tendrían entre 24 y 49 años de edad en la muestra considerada de Enaho 2014-2016. Se considera 
tales cohortes de individuos para identificar efectos causales en etapas crecientes de la curva salarios - edad. Los coeficientes $\eta_{t}$ representan el efecto de la desregulación en los determinantes que permitieron el acceso a estudios universitarios. La existencia del experimento natural permitiría dar cuenta de la la potencial correlación entre los factores inobservables (principalmente la habilidad) que determinan tanto los resultados laborales como el acceso a al educación universitaria.

En la estrategia empírica empleada en el presente estudio, en primer término se estimarán los efectos de forma reducida de la cohorte de nacimiento en los resultados laborales, para ello se reemplaza la ecuación (2) en la ecuación (1) de la siguiente forma:

$$
y_{i}=\alpha_{3}+\sum_{t=1980}^{1990} \beta_{t} d_{i, t}+\rho_{1}\left(c_{i}\right)+\rho_{2}\left(a_{i}\right)+X_{i}^{\prime} \lambda_{3}+\mu_{i, j}+\zeta_{i}
$$

En la ecuación anterior, $\alpha_{3}=\alpha_{1}+\delta\left(\alpha_{2}\right), \beta_{t}=\delta\left(\eta_{t}\right), \rho_{1}=\delta(\theta)+\alpha_{1}, \rho_{2}=\gamma_{2}$, $\rho_{3}=\delta+1, \lambda_{3}=\delta\left(\lambda_{2}\right)+\lambda_{1} \mathrm{y} \zeta_{i}=\delta\left(\varepsilon_{i}\right)+\nu_{i}$. En la estrategia empírica propuesta, si el acceso a la educación universitaria fue motivada por una mayor exposición a la desregulación y el coeficiente $\delta$ de la ecuación (1) es positivo, la variables dummies que representan las cohortes de nacimiento entre 1980 y 1990 deberían tener efectos positivos y significativos en el modelo de forma reducida (ecuación 3). El efecto de la educación universitaria en los resultados laboral será identificado usando las dummies de cohorte de nacimiento como instrumentos de la educación en una aproximación por IV. En esta aproximación, se asume que los instrumentos sólo tienen efectos en los resultados laborales a través de incrementar el acceso (años de educación) a la educación universitaria.

El efecto de la educación superior en universidades entrantes en los resultados laborales está representada en la ecuación (1), donde el coeficiente $\delta$ representa el verdadero efecto causal de la educación superior en los resultados laborales. Dicho coeficiente puede ser interpretado como el incremento promedio en los resultados de interés si una unidad adicional de educación superior fuera asignada a una muestra aleatoria de la población. Si bien es posible estimar empíricamente el coeficiente $\delta$ a través del estimador OLS, asumiendo que la educación $\left(s_{i}\right)$ es ortogonal (o no está correlacionada) tanto a los determinantes no observables de los resultados laborales $\left(\varepsilon_{i}\right)$ como los determinantes observables $\left(X_{i}\right)$, en la literatura empírica se ha discutido ampliamente sobre las posibles fuentes de sesgos en el estimador OLS [Griliches 1977] y [Card 1999].

Entre las principales fuentes de sesgos, se ha discutido el sesgo por variable omitida, en la cual el coeficiente estimado por OLS $\left(\delta_{\text {ols }}\right)$ esta correlacionado positivamente con las características no observables (principalmente habilidad no observable) que determinan tanto la educación como los resultados laborales (lo cual se reflejaría en la correlación entre $\varepsilon_{i}$ y $\nu_{i}$ ). Si el sesgo por habilidad es positivo la estimación por 
OLS del coeficiente $\delta$ sobre-estimaría la relación entre educación y resultados laborales $\left(\delta_{o l s}>\delta\right)$. Otra fuente de sesgo en OLS, es el sesgo generado por error de medición en los regresores, principalmente, en la medición de la educación superior observada. El error de medición puede ser generado por errores en las preguntas sobre educación aplicadas en las encuestas a individuos o por la heterogeneidad en la calidad educativa de las universidades, este sesgo es denominado usualmente como sesgo de atenuación $\left(\delta_{\text {ols }}<\delta\right)$.

La aproximación por variables instrumentales (IV) es la solución estándar que se ha empleado en la literatura para solucionar los sesgos por variable omitida y de atenuación ([Card 1999]). Se asume la existencia de un vector de instrumentos $\left(d_{i}\right)$ que esta correlacionado con la educación $\left(s_{i}\right)$ pero no correlacionado con cualquier otro determinante (observable o inobservable) de los resultados laborales $\left(y_{i}\right)$, este supuesto constituye las restricciones de exclusión en la metodología IV.

Para el presente estudio, la aproximación por IV está planteada en las ecuaciones (1) y (2) correspondientes a un modelo sobreidentificado o de múltiples instrumentos, ambas ecuaciones constituyen la segunda y primera etapa, respectivamente, del estimador OLS de dos etapas (2SLS, por sus siglas en inglés). En la primera etapa se descompone el regresor endógeno en un componente exógeno y otro endógeno, en la segunda etapa se emplea el componente exógeno como determinante de la variable dependiente de interés, en la metodología IV-2SLS los múltiples instrumentos pueden ser combinados en un sólo índice ponderado ([Angrist e Imbens 1995]).

Se utiliza un vector de dummies por cohorte de nacimiento como determinantes de la probabilidad y años de educación superior en universidades entrantes, asumiendo que cohortes más jóvenes estarían más expuestas al proceso de desregulación del sistema universitario que genero un shock en la oferta de universidades. Si el proceso de desregulación ha tenido suficientes efectos de equilibrio general en los retornos a la educación superior, principalmente, en las cohortes de trabajadores nacidos después de 1980, estos efectos podrían estar representados en la estimación del coeficiente $\delta_{i v}$.

La metodología IV-2SLS ofrecería una estimación del Local Average Treatment Effects (LATE) cuando se considera la variable tratamiento $\left(s_{i}\right)$ como una variable binaria y del Average Causal Effects (ACE), el cual es una generalización del LATE cuando se considera el tratamiento como una variable no binaria relacionado hetereogenidad en el tratamiento en términos de distintos niveles de intensidad del tratamiento en términos de años de educación universitaria.

En términos del Modelo de Resultados Potenciales de [Rubin 1974], el resultado laboral observado es $y=s y_{1}+(1-s) y_{0}$, al ser la eduación $s$ un regresor endógeno, se asume que existe un instrumento binario $d$ que determina la educación de la forma: $s=d s_{1}+(1-d) s_{0}$. El efecto causal de tener estudios universitarios para un nivel del 
instrumento es $y_{1, d}-y_{0, d} ;$ mientras que el efecto causal de la exposición al instrumento para un nivel educativo es $y_{s, 1}-y_{s, 0}$, entonces el instrumento $(d)$ generaría una cadena causal que afecta a la educación $(s)$ y esta a su vez afecta a los resultados laborales $(y)$; es decir $d_{i}, s_{i}=s_{i}\left(d_{i}\right)$ y $y_{i}=y_{i}\left[s_{i}\left(d_{i}\right)\right]$.

Para que los estimados de LATE y ACE puedan ser interpretados como los efectos causales del acceso a educación universitaria y de los años de educación universitaria, respectivamente, se asume que (Teorema LATE: condiciones de validez de los instrumentos, ([Imbens y Angrist 1994]): (i) Supuesto de independencia, SUTVA ${ }^{4}$, (ii) Restricciones de exclusión, (iii) Existencia de la primera etapa, (iv) Monotonicidad.

Como se mencionó anteriormente, los múltiples instrumentos en la metodología IV2SLS pueden ser combinados en un sólo índice ponderado tanto para el estimado LATE como ACE, de acuerdo con ([Angrist e Imbens 1995]):

$$
\delta_{i v}^{l a t e}=\frac{\operatorname{cov}\left(y_{i}, g\left(d_{i}\right)\right)}{\operatorname{cov}\left(s_{i}, g\left(d_{i}\right)\right)}=\sum_{t=1}^{T} \lambda_{t} \delta_{d_{t}, d_{t-1}}
$$

En la ecuación anterior $\delta_{i v}^{l a t e}$ es el efecto causal (promedio ponderado) para todos aquellos individuos cuyo estatus de tratamiento (acceso a la educación universitaria) es afectado por el vector de instrumentos (cumplidores) ${ }^{5}$. El vector de instrumentos es: $d=d_{1}, d_{2}, . ., d_{T}$. Los ponderadores $\lambda_{t}$ para cada instrumento t se definen como:

$$
\lambda_{t}=\frac{\left(p\left(d_{t}\right)-p\left(d_{t-1}\right)\right) \sum_{l=1}^{T} \pi_{l}\left(g\left(d_{l}\right)\right)-E\left[g\left(d_{i}\right)\right]}{\left(\sum_{t=1}^{T}\left(p\left(d_{t}\right)-p\left(d_{t-1}\right)\right)\right)\left(\sum_{l=1}^{T} \pi_{l}\left(g\left(d_{l}\right)\right)-E\left[g\left(d_{i}\right)\right]\right)}
$$

Donde, $0 \leq \lambda_{t} \leq 1$ y $\sum_{l=1}^{T} \lambda_{t}=1, p_{d}=E\left[s_{i} \mid d_{i}=z\right]$ y $\pi_{l}=\operatorname{Pr}\left[d_{i}=d_{t}\right]$. Por su parte el índice ponderado para el estimado ACE para múltiples instrumentos y tratamiento no binario puede ser expresado como ([Angrist e Imbens 1995]):

$$
\delta_{i v}^{a c e}=\frac{E[Y(E[s \mid d]-E[s])]}{E[E[s \mid d](E[s \mid d]-E[s])]}=\sum_{t=1}^{T} \mu_{t} \delta_{d_{t}, d_{t-1}}
$$

En la ecuación anterior $\delta_{i v}^{a c e}$ es la respuesta causal (promedio ponderada) ante un cambio en la intensidad del tratamiento (años de educación universitaria de similar calidad) para todos aquellos individuos afectados por el vector de instrumentos (cumplidores). El vector de instrumentos es: $d=d_{1}, d_{2}, . ., d_{T}$. Los ponderadores $\mu_{t}$ para cada instrumento t se definen como:

\footnotetext{
${ }^{4}$ Stable Unit Treatment Values ([Holland 1986])

${ }^{5}$ LATE no es informativo sobre efectos de la educación en los resultados laborales para individuos cuyo nivel educativo no cambia debido a la exposición o no exposición al instrumento: nunca tomadores, siempre tomadores, desafiantes.
} 


$$
\mu_{t}=(E[s \mid d=t]-E[s \mid d=t-1]) \frac{\sum_{l=t}^{T}(E[s \mid d=l]-E[s])}{\sum_{l=0}^{T} \pi_{l} E[s \mid d=l](E[s \mid d=l]-E[s])}
$$

Donde, $0 \leq \mu_{t} \leq 1$ y $\sum_{l=1}^{T} \mu_{t}=1$ y $\pi_{l}=\operatorname{Pr}\left[d=d_{l}\right]$.

\section{Resultados}

\subsection{Descripción de los datos}

La información de las variables consideradas en el presente estudio provienen de la Encuesta Nacional de hogares (Enaho) correspondientes a los años 2014, 2015 y 2016. En dicha base de datos, se releva información de alrededor 91 mil individuos por año, de los cuales 66 mil pertenecen a la Población Económicamente Activa (PEA) y 64 mil son trabajadores ocupados en algún tipo de empleo. Este estudio se concentra en trabajadores urbanos, ocupados y asalariados. Como se observó en la Figura 4, los grupos de trabajadores mayormente expuestos a la desregulación del sistema universitario fueron aquellos individuos con secundario y estudios universitarios en universidades entrantes. Sobre ambos grupos, la Enaho releva información anual de más de 12 mil trabajadores asalariados, los cuales representan $38 \%$ de la PEA y el $40 \%$ de los trabajadores ocupados; el resto de ocupados son considerados en Enaho como empleadores, autoempleados o cuenta propistas, trabajadores del hogar y trabajadores familiares no remunerados.

En el Cuadro 1, se presentan las medias muestrales del grupo de trabajadores en estudio, el cual está conformado por individuos ocupados y asalariados en áreas urbanas nacidos entre los años 1965 y 1980. En el análisis econométrico, se consideró solamente trabajadores cuyas edades se encuentran entre 24 y 49 años, quiénes se encuentran ubicados en las etapas crecientes de la curva salarios-edad; y cuyos niveles educativos son secundario o universitario (universidad entrante).

En términos de resultados laborales por niveles educativos (columnas 2 y 3 del Cuadro 1), los salarios e ingresos anuales son mayores para trabajadores con mayor calificación, la proporción de trabajadores con condiciones informales de empleo es menor en trabajadores más calificados. Por su parte, en términos de características individuales, los trabajadores con estudios superiores en universidades entrantes son relativamente más jóvenes y mayoritariamente varones, la mayor proporción de trabajadores de origen indígena (aproximado a través de la lengua quechua o aymara) se concentra en el nivel secundario. Los trabajadores más calificados tienen en promedio 5 años más de educación. 
En términos de resultados laborales por cohortes de nacimiento que determinan los grupos de control y tratamiento (columnas 4 y 5 del Cuadro 1, respectivamente), los salarios e ingresos anuales son relativamente similares entre individuos de la cohorte más joven o grupo de tratamiento (nacidos entre 1980 y 1990) y la cohorte de referencia o grupo de control (nacidos entre 1965 y 1979), la proporción de trabajadores con condiciones informales de empleo es mayor en trabajadores más jóvenes. Por su parte, en términos de características individuales, la proporción de mujeres se incrementa en la cohorte de trabajadores más jóvenes y dicha cohorte se reduce la proporción de trabajadores de origen indígena. Los trabajadores más jóvenes tienen en promedio 0.4 años más de educación.

Cuadro 1: Medias muestrales por niveles educativos y cohortes de nacimiento

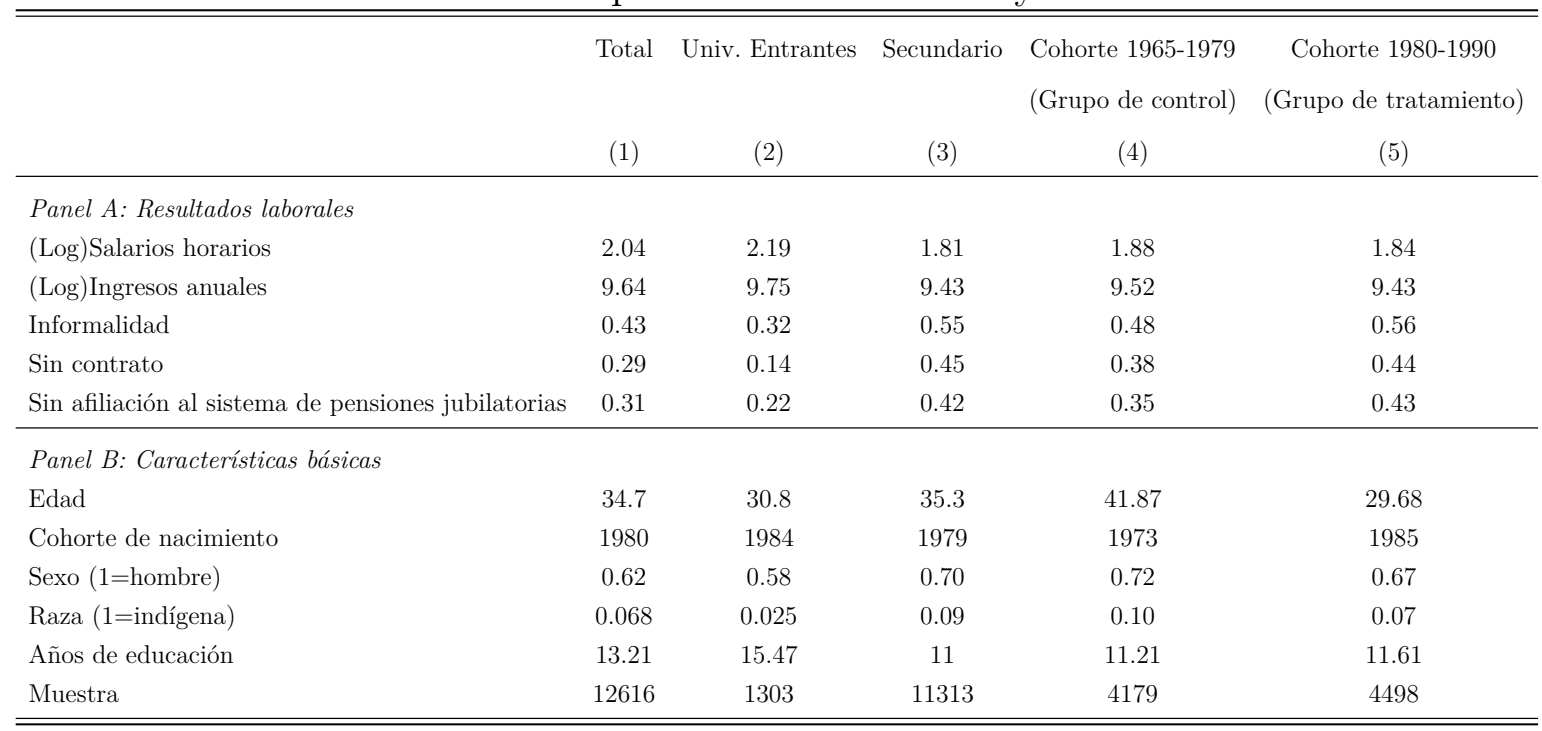

Muestra de individuos en áreas urbanas, ocupados, asalariados nacidos entre los años 1965 y 1990. Informalidad: proporción de trabajadores que no cuentan con ningún seguro de salud, ni cobertura de pensiones. Fuente: Enaho 2014-2016.

\subsection{Efectos de la desregulación en resultados laborales}

\subsubsection{Resultados I: Modelo de forma reducida}

Los resultados presentados en el Cuadro 2 provienen de la estimación por OLS de la ecuación (3) considerando individuos con estudios superiores en universidades entrantes e individuos con secundario, las columnas (1) y (2) muestran los coeficientes estimados asociados a las variables dummies por cohorte de nacimiento entre $1980 \mathrm{y}$ 1990 que capturarían el efecto de la cohorte de nacimiento tanto en la probabilidad de estudios universitarios (columna 1) en en los años de educación universitaria (columna 2). 
Controlando por una tendencia en la cohorte de nacimiento y la edad de los trabajadores, en ambas columnas los coeficientes estimados son positivos y significativos a partir de la cohorte de individuos nacidos en 1984 en adelante (la mayoría de coeficientes son significativos al 1\%); por su parte, la Prueba F sugiere que conjuntamente las dummies de cohorte de nacimiento son estadísticamente distintas de cero. En general, los coeficientes estimados se incrementan para cohortes más jóvenes, lo cual brindaría evidencia a favor del supuesto que la exposición a la desregulación del sistema universitario es una función creciente del año de nacimiento. Los resultados sugieren que para la cohorte de individuos nacidos en 1990 la desregulación habría incrementado en 16.6 puntos porcentuales la probabilidad de acceder a estudios universitarios e incrementado en 0.745 sus años de educación.

Las columnas (3) y (4) del Cuadro 2 se muestran similares estimaciones considerando como variables dependientes el (Log) del salario horario y la probabilidad de informalidad. Los resultados muestran efectos significativos de la cohorte nacimiento tanto en términos de premios salarias como en mejoras en las condiciones laborales; particular, se observa premios salariales por mayor calificación entre $9 \%$ y $17 \%$ para individuos nacidos en las cohortes más jóvenes y reducciones entre $9 \%$ y $11 \%$ en la probabilidad de estar en empleos informales.

En la Figura 5, los coeficientes estimados $\beta_{t}$ de la ecuación de forma reducida (ecuación 3) fueron graficados considerando como variables dependientes los años de educación universitaria, (Log) salarios horarios y la probabilidad de informalidad. Los coeficientes asociados a las dummies de nacimiento y las tres variables dependientes oscilan en torno a cero hasta las cohortes de individuos nacidos hasta 1979. Los coeficientes asociados a las dummies de nacimiento con la educación y los salarios se incrementan a partir de la cohorte de individuos nacidos en 1980; mientras que, la probabilidad de informalidad se reduce ligeramente a partir de dicha cohorte de nacimiento. En la estrategia de identificación propuesta se asume que la desregulación del sistema universitario sólo tendría efectos en los resultados laborales de interés a través de incrementar la probabilidad de acceder a estudios universitarios, incrementando los años de educación superior, dicha Figura valida en cierto modo dicho supuesto. 
Cuadro 2: Efectos de la cohorte de nacimiento en educación y resultados laborales

\begin{tabular}{|c|c|c|c|c|}
\hline & \multicolumn{2}{|c|}{ Educación } & \multicolumn{2}{|c|}{ Resultados laborales } \\
\hline & Probabilidad de estudios universitarios & Años de educación universitaria & $(\mathrm{Log})$ Salarios horarios & Probabilidad de informalidad \\
\hline & $(1)$ & $(2)$ & $(3)$ & $(4)$ \\
\hline \multirow[t]{2}{*}{1980} & $0.0416^{*}$ & $0.184^{*}$ & 0.0350 & -0.0421 \\
\hline & $(0.0219)$ & $(0.099)$ & $(0.0324)$ & $(0.0403)$ \\
\hline \multirow[t]{2}{*}{1981} & $0.0322^{*}$ & $0.151^{*}$ & $0.107^{* * *}$ & -0.00519 \\
\hline & $(0.0193)$ & $(0.088)$ & $(0.0406)$ & $(0.0410)$ \\
\hline \multirow[t]{2}{*}{1982} & $0.0481^{* *}$ & $0.219^{* * *}$ & $0.0721^{* *}$ & $-0.0635^{*}$ \\
\hline & $(0.0175)$ & $(0.0793)$ & $(0.0321)$ & $(0.0335)$ \\
\hline \multirow[t]{2}{*}{1983} & $0.0350^{* *}$ & $0.164^{* *}$ & $0.0924^{* *}$ & -0.0544 \\
\hline & $(0.0164)$ & $(0.0749)$ & $(0.0371)$ & $(0.0406)$ \\
\hline \multirow[t]{2}{*}{1984} & $0.0606^{* * *}$ & $0.288^{* * *}$ & 0.0387 & -0.0107 \\
\hline & $(0.0225)$ & $(0.102)$ & $(0.0413)$ & $(0.0492)$ \\
\hline \multirow[t]{2}{*}{1985} & $0.0807^{* * *}$ & $0.387^{* * *}$ & $0.0688^{*}$ & $-0.0690^{*}$ \\
\hline & $(0.0236)$ & $(0.112)$ & $(0.0363)$ & $(0.0412)$ \\
\hline \multirow[t]{2}{*}{1986} & $0.0840^{* * *}$ & $0.388^{* * *}$ & 0.0667 & -0.0101 \\
\hline & $(0.0233)$ & $(0.107)$ & $(0.0424)$ & $(0.0398)$ \\
\hline \multirow[t]{2}{*}{1987} & $0.125^{* * *}$ & $0.556^{* * *}$ & $0.0919^{* *}$ & $-0.0888^{*}$ \\
\hline & $(0.0283)$ & $(0.127)$ & $(0.0415)$ & $(0.0461)$ \\
\hline \multirow[t]{2}{*}{1988} & $0.132^{* * *}$ & $0.570^{* * *}$ & $0.104^{* *}$ & $-0.113^{* *}$ \\
\hline & $(0.0297)$ & $(0.128)$ & $(0.0431)$ & $(0.0474)$ \\
\hline \multirow[t]{2}{*}{1989} & $0.153^{* * *}$ & $0.696^{* * *}$ & $0.129^{* * *}$ & -0.0507 \\
\hline & $(0.0301)$ & $(0.131)$ & $(0.0474)$ & $(0.0463)$ \\
\hline \multirow[t]{2}{*}{1990} & $0.186^{* * *}$ & $0.842^{* * *}$ & $0.166^{* * *}$ & $-0.102^{* *}$ \\
\hline & $(0.0310)$ & $(0.138)$ & $(0.0480)$ & $(0.0495)$ \\
\hline \multirow[t]{2}{*}{ Cohort-trend } & 0.0026 & 0.0137 & $0.0356^{* *}$ & 0.00536 \\
\hline & $(0.0087)$ & $(0.0405)$ & $(0.0148)$ & $(0.0175)$ \\
\hline \multirow[t]{2}{*}{ Edad } & 0.0028 & 0.0152 & $0.0460 * * *$ & -0.00525 \\
\hline & $(0.0088)$ & $(0.0410)$ & $(0.0149)$ & $(0.0177)$ \\
\hline F-statistics & 12.46 & 11.83 & 1.54 & 1.21 \\
\hline Observations & 8,677 & 8,678 & 8,677 & 9,795 \\
\hline R-squared & 0.061 & 0.060 & 0.083 & 0.051 \\
\hline
\end{tabular}

Todas las especificaciones incluyen como controles sexo, raza y efectos fijos a nivel de región de residencia.

El grupo de control está constituido por individuos pertenecientes a las cohortes entre 1965 y 1979.

Probabilidad de informalidad: probabilidad que los trabajadores que no cuenten con ningún seguro de salud, ni cobertura de pensiones.

La prueba $\mathrm{F}$ evalúa la hipótesis nula que los coeficientes asociados a las dummies de cohortes de nacimiento son conjuntamente iguales a cero.

Clustered Standard errors in parentheses.

*** $\mathrm{p}<0.01, * * \mathrm{p}<0.05,{ }^{*} \mathrm{p}<0.1$. 
Figura 5: Coeficientes de los años de nacimiento en las ecuaciones de forma reducida de educación universitaria, salarios e informalidad

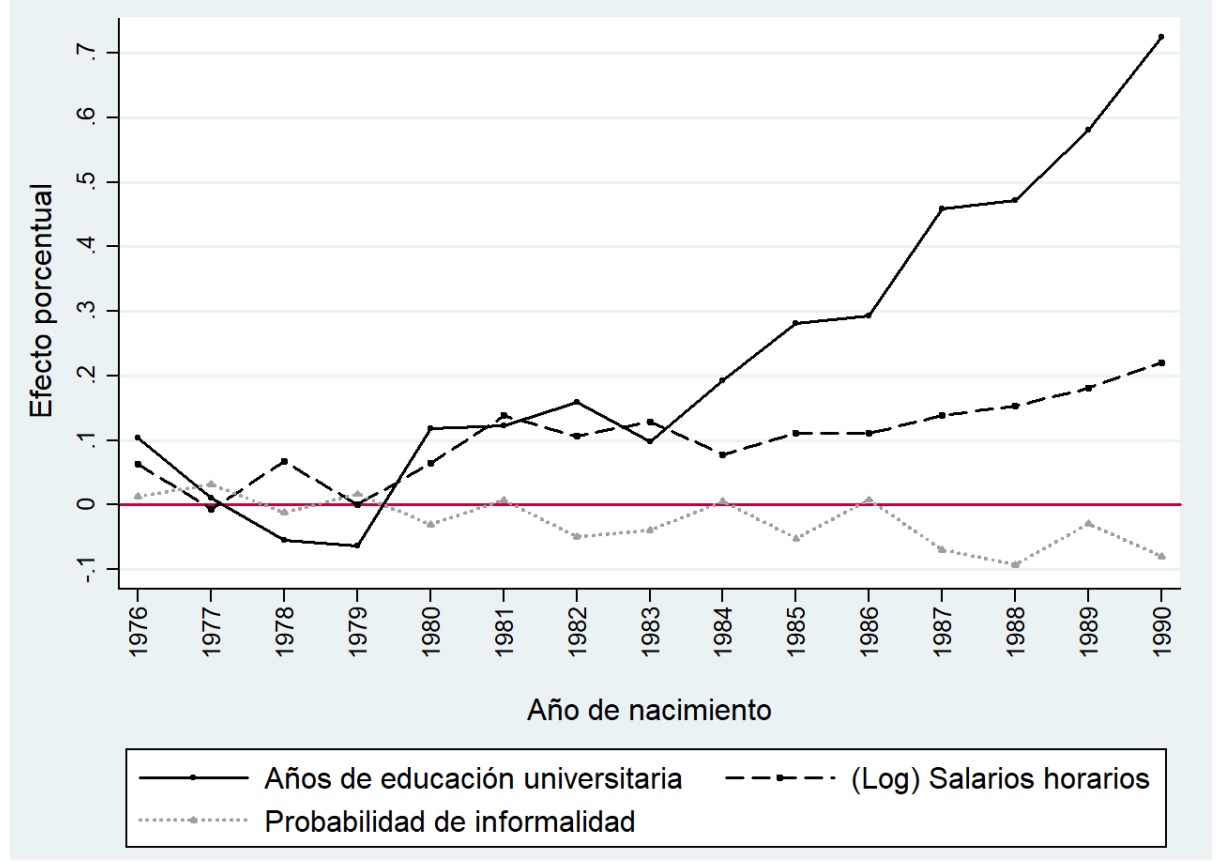

Elaboración Propia.

\subsubsection{Resultados II: Modelo de Variables Instrumentales}

Algunas de las condiciones de validez de los instrumentos pueden ser evaluadas o verificadas empíricamente. En primer término, en en el Cuadro 9 del Apéndice se muestra la primera etapa de la metodología IV-2SLS planteada en la ecuación (2), básicamente se busca verificar la relevancia del vector de instrumentos para explicar tanto la probabilidad de estudios universitarios como los años de educación universitaria, en ambos casos se encuentra que la mayoría de dummies por cohorte de nacimiento son significativas al $1 \%$, la prueba-F de significancia conjunta de los instrumentos resultó 12.46 y 11.83 , respectivamente ${ }^{6}$.

De acuerdo con [Stock y Yogo 2002], cuando los instrumentos no tienen suficiente significancia estadística para explicar cambios en el regresor endógeno serían considerados como instrumentos débiles, lo cual produciría que el estimador IV sea sesgado. Los autores proponen operativamente valores críticos que permitan en función del valor de la prueba-F resultante de la primera etapa determinar si los instrumentos considerados son débiles ${ }^{7}$; en particular, considerando un regresor endógeno, un nivel máximo de sesgo deseado en la estimación IV relativa a la estimación OLS de $10 \%$ y 11 instru-

\footnotetext{
${ }^{6}$ La prueba-F plantea la hipótesis nula que los coeficientes asociados a las variables dummies por cohorte de nacimiento son conjuntamente cero, con los resultados obtenidos no es posible aceptar dicha hipótesis nula.

${ }^{7}$ Ver Tabla 1 de dicha referencia.
} 
mentos, el valor crítico definido es 11.51; los resultados de la prueba-F presentados en en el Cuadro 9 del Apéndice sugieren que las dummies por cohorte de nacimiento empleadas en la estimación IV podrían no ser consideradas conjuntamente como instrumentos débiles. Por su parte, en el Cuadro 10 del Apéndice, se presentan un conjunto de resultados de pruebas de especificación de las estimaciones IV, con el objetivo de verificar de algún modo las propiedades necesarias, restricciones de exclusión, para que las estimaciones LATE y ACE puedan ser consideradas plausibles de efectos causales.

En el Cuadro 3 se presentan una serie de estimaciones por OLS y IV-2SLS del efecto de la educación superior en los resultados laborales de interés, para todas las estimaciones se consideraron individuos nacidos entre 1965 y 1990 y todas las especificaciones incluyen una tendencia por cohorte de nacimiento, edad de los individuos y covariables de control (sexo, raza y efectos fijos a nivel de región de residencia). Las columnas numeradas impares presentan las estimaciones por OLS; mientras que las columnas pares las estimaciones por IV-2SLS en las cuales se emplean como instrumentos variables dummies por cada cohorte de nacimiento entre 1980 y 1990 (el grupo de control son individuos nacidos en las cohortes entre 1965 y 1979). En el Panel A se considera la variable de educación $\left(s_{i}\right)$ como una variable binaria igual a 1 si el individuo i tiene estudios superiores en universidades entrantes y 0 si tiene secundario; mientras que el panel B presenta similares resultados considerando la educación como los años de estudios universitarios en universidades entrantes.

Respecto del efecto causal del acceso a estudios universitarios (LATE), los resultados del estimador IV sugieren que trabajadores con estudios superiores tienen mayores niveles de ingresos laborales y mejores condiciones laborales; en particular, tienen un premio salarial de 59.5 puntos porcentuales respecto de trabajadores con estudios secundarios, el coeficiente que asocia la educación con los (Log)Ingresos anuales si bien es positivo no resultó significativo estadísticamente. Los resultados sobre las condiciones laborales sugieren que los estudios universitarios reducen en 52 puntos porcentuales la probabilidad de informalidad en el empleo respecto de trabajadores con educación secundaria; en particular, reduce en 57.9 puntos porcentuales la probabilidad de no tener contrato de trabajo, el efecto estimado en la probabilidad de no tener afiliación al sistema de pensiones jubilatorias si bien es negativo no resultó significativo estadísticamente. Los dos últimos resultados sugieren que el acceso a estudios universitarios no mejora necesariamente todas las dimensiones de informalidad de los trabajadores para el caso peruano.

Respecto de la respuesta causal los años de estudios universitarios (ACE), los resultados del estimador IV sugieren que por cada año adicional de estudios superiores los salarios se incrementan en 13.5 puntos porcentuales y se reduciría la probabilidad de informalidad en el empleo en 11.3 puntos porcentuales. Tales resultados son cualitati- 
vamente similares cuando la educación es aproximada a través de una variable binaria que representa el acceso a estudios universitarios. De similar modo, el efecto estimado sobre la probabilidad de no tener afiliación al sistema de pensiones jubilatorias no resultó significativo estadísticamente, el resultado significativo sugiere que por cada año de educación universitaria la probabilidad de no tener contrato laboral se reduce en 13 puntos porcentuales.

En general, en todas las estimaciones por OLS e IV que resultaron significativas estadísticamente, los coeficientes estimados por IV-2SLS resultaron mayores que los estimados por OLS $\left(\delta_{i v-2 s l s}>\delta_{\text {ols }}\right)$, lo cual sugiere la posibilidad de la presencia del sesgo de atenuación en las estimación por OLS generadas, probablemente, por errores de medición en la educación, este resultado es consiste con una gran número de estudios disponibles en la literatura sistematizados en [Card 1999] $]^{8}$. Otra posible explicación implica que el efecto de la educación universitaria es heterogénea y varía entre individuos, por ello las estimaciones por OLS e IV capturarían distintos puntos de la distribución de tal efecto ([Maurin y McNally 2008]), donde los individuos más expuestos a la desregulación tendrían relativamente mayores retornos marginales.

\footnotetext{
${ }^{8}$ En dicha referencia, en promedio la brecha estimada entre las estimaciones OLS e IV sería del $30 \%$, en el presente estudio se encuentra que dicha diferencia es en promedio $40 \%$, lo cual sugiere que el error de medición de la educación sería un problema particularmente relevante en la estrategia empírica propuesta
} 
Cuadro 3: Efectos de la educación en los resultados laborales por IV: segunda etapa, 2SLS

\begin{tabular}{|c|c|c|c|c|c|c|c|c|c|c|}
\hline & \multicolumn{4}{|c|}{ Ingresos laborales } & \multicolumn{6}{|c|}{ Condiciones laborales } \\
\hline & \multicolumn{2}{|c|}{$(\mathrm{Log})$ Salarios horarios } & \multicolumn{2}{|c|}{$(\mathrm{Log})$ Ingresos anuales } & \multicolumn{2}{|c|}{ Informalidad } & \multicolumn{2}{|c|}{ Sin contrato } & \multicolumn{2}{|c|}{ Sin pensiones jubilatorias } \\
\hline & OLS & IV & OLS & IV & OLS & IV & OLS & IV & OLS & IV \\
\hline & (1) & $(2)$ & (3) & (4) & $(5)$ & (6) & (7) & (8) & (9) & $(10)$ \\
\hline \multicolumn{11}{|l|}{ Panel A: Acceso a estudios universitarios } \\
\hline Estudios universitarios (universidad entrante) & $\begin{array}{c}0.433^{* * *} \\
(0.0227)\end{array}$ & $\begin{array}{c}0.595^{* * *} \\
(0.201)\end{array}$ & $\begin{array}{c}0.417^{* * *} \\
(0.0316)\end{array}$ & $\begin{array}{c}0.368 \\
(0.273)\end{array}$ & $\begin{array}{c}-0.284^{* * *} \\
(0.0237)\end{array}$ & $\begin{array}{c}-0.520^{* *} \\
(0.242)\end{array}$ & $\begin{array}{c}-0.350 * * * \\
(0.0255)\end{array}$ & $\begin{array}{c}-0.579 * * \\
(0.249)\end{array}$ & $\begin{array}{c}-0.273^{* * *} \\
(0.0218)\end{array}$ & $\begin{array}{l}-0.239 \\
(0.262)\end{array}$ \\
\hline Cohort-trend & $\begin{array}{c}0.0374^{* * *} \\
(0.0130)\end{array}$ & $\begin{array}{c}0.0358^{* * *} \\
(0.0123)\end{array}$ & $\begin{array}{c}0.0376^{* * *} \\
(0.0133)\end{array}$ & $\begin{array}{c}0.0381^{* * *} \\
(0.0134)\end{array}$ & $\begin{array}{l}0.00389 \\
(0.0166)\end{array}$ & $\begin{array}{l}0.00608 \\
(0.0151)\end{array}$ & $\begin{array}{l}0.00359 \\
(0.0177)\end{array}$ & $\begin{array}{l}0.00573 \\
(0.0160)\end{array}$ & $\begin{array}{l}0.00965 \\
(0.0134)\end{array}$ & $\begin{array}{l}0.00933 \\
(0.0132)\end{array}$ \\
\hline Edad & $\begin{array}{c}0.0446^{* * *} \\
(0.0130)\end{array}$ & $\begin{array}{c}0.0442^{* * *} \\
(0.0124)\end{array}$ & $\begin{array}{c}0.0471^{* * *} \\
(0.0133)\end{array}$ & $\begin{array}{c}0.0473^{* * *} \\
(0.0133)\end{array}$ & $\begin{array}{l}-0.00446 \\
(0.0165)\end{array}$ & $\begin{array}{c}-0.00382 \\
(0.0153)\end{array}$ & $\begin{array}{l}-0.00360 \\
(0.0175)\end{array}$ & $\begin{array}{l}-0.00297 \\
(0.0161)\end{array}$ & $\begin{array}{l}0.00146 \\
(0.0132)\end{array}$ & $\begin{array}{l}0.00137 \\
(0.0132)\end{array}$ \\
\hline Observations & 8,677 & 8,677 & 9,719 & 9,719 & 9,795 & 9,795 & 9,795 & 9,795 & 9,795 & 9,795 \\
\hline R-squared & 0.156 & 0.145 & 0.179 & 0.178 & 0.075 & 0.057 & 0.085 & 0.068 & 0.086 & 0.085 \\
\hline \multicolumn{11}{|l|}{ Panel B: Años de estudios universitarios } \\
\hline Años de estudios universitarios (universidad entrante) & $\begin{array}{c}0.0979 * * * \\
(0.00492)\end{array}$ & $\begin{array}{c}0.135^{* * *} \\
(0.0450)\end{array}$ & $\begin{array}{c}0.0950^{* * *} \\
(0.00688)\end{array}$ & $\begin{array}{c}0.0876 \\
(0.0619)\end{array}$ & $\begin{array}{c}-0.0633^{* * *} \\
(0.00528)\end{array}$ & $\begin{array}{c}-0.113^{* *} \\
(0.0537)\end{array}$ & $\begin{array}{c}-0.0778^{* * *} \\
(0.00560)\end{array}$ & $\begin{array}{c}-0.128^{* *} \\
(0.0565)\end{array}$ & $\begin{array}{c}-0.0607^{* * *} \\
(0.00482)\end{array}$ & $\begin{array}{l}-0.0502 \\
(0.0593)\end{array}$ \\
\hline Cohort-trend & $\begin{array}{c}0.0371^{* * *} \\
(0.0129)\end{array}$ & $\begin{array}{c}0.0354^{* * *} \\
(0.0122)\end{array}$ & $\begin{array}{c}0.0373^{* * *} \\
(0.0133)\end{array}$ & $\begin{array}{c}0.0377^{* * *} \\
(0.0134)\end{array}$ & $\begin{array}{l}0.00401 \\
(0.0166)\end{array}$ & $\begin{array}{l}0.00620 \\
(0.0151)\end{array}$ & $\begin{array}{l}0.00374 \\
(0.0177)\end{array}$ & $\begin{array}{l}0.00593 \\
(0.0160)\end{array}$ & $\begin{array}{l}0.00976 \\
(0.0134)\end{array}$ & $\begin{array}{l}0.00931 \\
(0.0133)\end{array}$ \\
\hline Edad & $\begin{array}{c}0.0443^{* * *} \\
(0.0129)\end{array}$ & $\begin{array}{c}0.0438^{* * *} \\
(0.0123)\end{array}$ & $\begin{array}{c}0.0468^{* * *} \\
(0.0133)\end{array}$ & $\begin{array}{c}0.0469 * * * \\
(0.0133)\end{array}$ & $\begin{array}{l}-0.00429 \\
(0.0165)\end{array}$ & $\begin{array}{c}-0.00356 \\
(0.0153)\end{array}$ & $\begin{array}{l}-0.00339 \\
(0.0175)\end{array}$ & $\begin{array}{c}-0.00266 \\
(0.0161)\end{array}$ & $\begin{array}{l}0.00161 \\
(0.0132)\end{array}$ & $\begin{array}{l}0.00146 \\
(0.0133)\end{array}$ \\
\hline Observations & 8,677 & 8,677 & 9,719 & 9,719 & 9,795 & 9,795 & 9,795 & 9,795 & 9,795 & 9,795 \\
\hline R-squared & 0.160 & 0.148 & 0.181 & 0.181 & 0.075 & 0.059 & 0.086 & 0.069 & 0.086 & 0.085 \\
\hline
\end{tabular}

Instrumentos: variables dummies por cada cohorte de nacimiento entre 1980 y 1990.

El grupo de control está constituido por individuos pertenecientes a las cohortes entre 1965 y 1979.

Probabilidad de informalidad: probabilidad que los trabajadores que no cuenten con ningún seguro de salud, ni cobertura de pensiones.

Todas las especificaciones incluyen como controles sexo, raza y efectos fijos a nivel de región de residencia.

Clustered Standard errors in parentheses.

*** $\mathrm{p}<0.01, * * \mathrm{p}<0.05, * \mathrm{p}<0.1$. 


\subsubsection{Resultados III: Heterogeneidades}

Los trabajadores nacidos después de 1980 es la cohorte de individuos más afectados por la desregulación del sistema universitario peruano, debido al incremento en la oferta de universidades y la flexibilización en los requisitos de admisión a las universidades. Sin embargo, es posible que los trabajadores estén expuestos a la reforma de manera heterogénea, por ello en esta subsección se exploran posibles heterogenidades en los efectos en función a la variabilidad en la determinadas características socioeconómicas dentro de las cohortes de comparación. En particular se han comparado los resultados en base a diferencias en el sexo, raza y quintiles de ingreso per cápita familiar.

En las Figuras 6, 7 y 8 del Apéndice, se han graficado los coeficientes estimados $\beta_{t}$ de la ecuación de forma reducida (ecuación 3 ) considerando como variable dependiente los años de educación en función a los años de nacimiento desagregado por sexo, raza y quintiles de ingreso famliar per cápita, respectivamente. Tales figuras sugieren que la desregulación del sistema universitario incrementó los años de educación tanto de hombres como mujeres nacidos después de 1980, no obstante dicho efecto es mayor para el caso de las mujeres y para trabajadores de origen no indígena. Por su parte, el efecto del año de nacimiento en la escolaridad es relativamente mayor en quintiles mas altos del ingreso familiar per cápita. Dichas figuras sugieren que la desregulación tendría efectos en los resultados laborales a través de incrementar los años de educación superior, afectado de manera relativamente heterogénea a los individuos.

En el Cuadro 4 se presentan resultados de los estimados LATE y ACE para hombres, mujeres e indígenas y no-indígenas. La condición de indígena fue construida en función a la lengua materna de los individuos (lengua materna Quechua y Aymara). En primer término, los resultados del estimado ACE sugieren que la magnitud del efecto de la educación en los salarios sería mayor en mujeres respecto de hombres; las mujeres que acceden a estudios universitarios tienen en promedio un premio salarial 73 puntos porcentuales más alto respecto de mujeres con estudios secundarios, dicho premio salarial es 42 puntos porcentuales para hombres; en contraste, el efecto de la educación universitaria en la probabilidad de informalidad es cualitativamente mayor en hombres que en mujeres.

En segundo término, los resultados del estimado LATE sugieren d similar modo que la magnitud del efecto de los años de educación universitaria en los salarios sería mayor en mujeres respecto de hombres. Por cada año adicional de estudios las mujeres tienen, en promedio, un premio salarial de 16 puntos porcentuales más alto respecto de mujeres con estudios secundarios, dicho premio salarial es 9.3 puntos porcentuales para hombres, lo cual sugiere que el mercado laboral peruano valoraría mujeres más calificadas. En contraste, el efecto de los años adicionales de educación en la probabi- 
lidad de informalidad es mayor en hombres que en mujeres, lo anterior sugiere que si bien mujeres con estudios universitarios obtienen un mayor premio salarial respecto de aquellas menos calificadas, sus condiciones laborales son relativamente más informales que los hombres.

En tercer término, respecto de los estimados LATE y ACE por raza de los trabajadores, sólo se encuentran resultados significativos en salarios y condiciones laborales para aquellos individuos definidos como no-indígenas, en este grupo por cada año adicional de estudios universitarios los salarios se incrementan en 12.5 puntos porcentuales. Los efectos estimados para el grupo de trabajadores definidos como indígenas si bien es positivo y cualitativamente mayor que el otro grupo, los coeficientes estimados no resultaron significativos estadísticamente, esto podría ser explicado por el reducido número de observaciones, como se observó en el Cuadro 1 solamente el $7 \%$ de la muestra empleada se define como indígenas.

En el Cuadro 5 se presentan resultados de los estimados LATE y ACE por quintiles de ingreso familiar per-cápita. Los resultados del estimado LATE sugieren que el efecto de la educación en los salarios y la informalidad laboral es significativo estadísticamente para individuos en el quintil de ingresos más alto; los individuos con estudios universitarios en dicho quintil tienen en promedio un premio salarial 73 puntos porcentuales más alto respecto de individuos con estudios secundarios; Por su parte, los individuos en el quintil más alto de ingresos con estudios universitarios tienen una menor probabilidad de informalidad respecto de individuos con estudios secundarios.

Respecto de los resultados del estimado ACE, el efecto de los años de educación en los salarios y la informalidad laboral es significativo estadísticamente para individuos en el quintil de ingresos más alto; en este quintil por cada año adicional de estudios universitarios los salarios se incrementan en 16 puntos porcentuales; mientras que la probabilidad de informalidad laboral se reduce en 15 puntos porcentuales. 
Cuadro 4: Efectos heterogéneos de la educación en los resultados laborales por sexo y raza: segunda etapa, IV-2SLS

\begin{tabular}{|c|c|c|c|c|c|c|c|c|}
\hline & \multicolumn{4}{|c|}{ Por sexo } & \multicolumn{4}{|c|}{ Por raza } \\
\hline & \multicolumn{2}{|c|}{ (Log)Salarios horarios } & \multicolumn{2}{|c|}{ Informalidad } & \multicolumn{2}{|c|}{ (Log)Salarios horarios } & \multicolumn{2}{|c|}{ Informalidad } \\
\hline & Hombre & Mujer & Hombre & Mujer & Indigena & No-indigena & Indigena & No-indigena \\
\hline & (1) & $(2)$ & (3) & (4) & (5) & (6) & (7) & (8) \\
\hline \multicolumn{9}{|l|}{ Panel A: Acceso a estudios universitarios } \\
\hline \multirow[t]{2}{*}{$\delta_{i v}^{\text {late }}:$ Estudios universitarios (universidad entrante) } & $0.417^{*}$ & $0.731^{* * *}$ & $-0.646^{* *}$ & $-0.430^{*}$ & 0.986 & $0.665^{* * *}$ & -0.0872 & $-0.566^{* *}$ \\
\hline & $(0.217)$ & $(0.204)$ & $(0.259)$ & $(0.252)$ & $(0.780)$ & $(0.211)$ & $(0.676)$ & $(0.250)$ \\
\hline Observations & 5,918 & 2,759 & 6,668 & 3,127 & 755 & 7,922 & 874 & 8,921 \\
\hline R-squared & 0.109 & 0.153 & 0.028 & 0.067 & 0.196 & 0.130 & 0.139 & 0.038 \\
\hline \multicolumn{9}{|l|}{ Panel B: Años de estudios universitarios } \\
\hline \multirow[t]{2}{*}{$\delta_{i v}^{a c e}:$ Años de estudios universitarios (universidad entrante) } & $0.0935^{*}$ & $0.159^{* * *}$ & $-0.135^{* *}$ & $-0.0982^{*}$ & 0.235 & $0.152^{* * *}$ & -0.0140 & $-0.125^{* *}$ \\
\hline & $(0.0479)$ & $(0.0447)$ & $(0.0545)$ & $(0.0555)$ & $(0.169)$ & $(0.0474)$ & $(0.151)$ & $(0.0556)$ \\
\hline Observations & 5,918 & 2,759 & 6,668 & 3,127 & 755 & 7,922 & 874 & 8,921 \\
\hline R-squared & 0.113 & 0.158 & 0.036 & 0.065 & 0.197 & 0.131 & 0.137 & 0.038 \\
\hline
\end{tabular}

Instrumentos: variables dummies por cada cohorte de nacimiento entre 1980 y 1990.

El grupo de control está constituido por individuos pertenecientes a las cohortes entre 1965 y 1979.

Probabilidad de informalidad: probabilidad que los trabajadores que no cuenten con ningún seguro de salud, ni cobertura de pensiones.

Todas las especificaciones incluyen efectos fijos a nivel de región de residencia.

Clustered Standard errors in parentheses.

$* * * \mathrm{p}<0.01,{ }^{* *} \mathrm{p}<0.05,{ }^{*} \mathrm{p}<0.1$. 
Cuadro 5: Efectos heterogéneos de la educación en los resultados laborales por quintiles del ingreso familiar per-cápita: segunda etapa,

\begin{tabular}{|c|c|c|c|c|c|c|c|c|c|c|}
\hline & \multicolumn{5}{|c|}{ (Log)Salarios horarios } & \multicolumn{5}{|c|}{ Informalidad } \\
\hline & Quintil 1 & Quintil 2 & Quintil 3 & Quintil 4 & Quintil 5 & Quintil 1 & Quintil 2 & Quintil 3 & Quintil 4 & Quintil 5 \\
\hline & $(1)$ & $(2)$ & $(3)$ & $(4)$ & $(5)$ & $(6)$ & $(7)$ & $(8)$ & $(9)$ & $(10)$ \\
\hline \multicolumn{11}{|l|}{ Panel A: Acceso a estudios universitarios } \\
\hline \multirow{2}{*}{$\delta_{i v}^{\text {late }}:$ Estudios universitarios (universidad entrante) } & -0.269 & -1.046 & -0.264 & 0.145 & $0.734^{* *}$ & -0.717 & 0.523 & 0.558 & -0.238 & $-0.778^{* *}$ \\
\hline & $(0.527)$ & $(0.519)$ & $(0.273)$ & $(0.189)$ & $(0.315)$ & $(0.683)$ & $(0.804)$ & $(0.509)$ & $(0.245)$ & $(0.373)$ \\
\hline Observations & 2,148 & 2,153 & 1,841 & 1,530 & 1,005 & 2,423 & 2,422 & 2,069 & 1,719 & 1,162 \\
\hline R-squared & 0.113 & 0.05 & 0.030 & 0.120 & 0.013 & 0.069 & 0.070 & 0.071 & 0.051 & 0.052 \\
\hline \multicolumn{11}{|l|}{ Panel B: Años de estudios universitarios } \\
\hline \multirow[t]{2}{*}{$\delta_{i v}^{a c e}:$ Años de estudios universitarios (universidad entrante) } & -0.0563 & -0.253 & -0.0539 & 0.0201 & $0.157^{* *}$ & -0.152 & 0.111 & 0.128 & -0.0525 & $-0.151^{* *}$ \\
\hline & $(0.121)$ & $(0.120)$ & $(0.0624)$ & $(0.0438)$ & $(0.0660)$ & $(0.154)$ & $(0.187)$ & $(0.117)$ & $(0.0571)$ & $(0.0741)$ \\
\hline Observations & 2,148 & 2,153 & 1,841 & 1,530 & 1,005 & 2,423 & 2,422 & 2,069 & 1,719 & 1,162 \\
\hline R-squared & 0.115 & 0.116 & 0.035 & 0.111 & 0.028 & 0.073 & 0.074 & 0.075 & 0.051 & 0.052 \\
\hline
\end{tabular}

Instrumentos: variables dummies por cada cohorte de nacimiento entre 1980 y 1990.

El grupo de control está constituido por individuos pertenecientes a las cohortes entre 1965 y 1979.

Probabilidad de informalidad: probabilidad que los trabajadores que no cuenten con ningún seguro de salud, ni cobertura de pensiones.

Todas las especificaciones incluyen efectos fijos a nivel de región de residencia.

Clustered Standard errors in parentheses.

$* * * \mathrm{p}<0.01, * * \mathrm{p}<0.05, * \mathrm{p}<0.1$. 


\subsection{Pruebas de robustez: experimentos falsos}

¿Qué tan válidos son los estimados LATE y ACE?, los resultados presentados en la subsección anterior comparan el efecto de la educación universitaria entre individuos con estudios superiores en universidades entrantes respecto de individuos con estudios secundarios. Se asume que ambos grupos serían los mayormente afectados por el proceso de desregulación del sistema universitario, donde el grado de exposición es una función creciente del año de nacimiento. Se asumió que, de no ser por el proceso de desregulación, los individuos con estudios secundarios no habrían podido acceder al sistema universitario, dado que la universidades entrantes muestran en general menores niveles de selectividad en el proceso de admisión de estudiantes. Con ello se asumió que el nivel de habilidad inobservables entre ambos grupos sería en promedio similar.

El supuesto de identificación implica que el año de nacimiento podría incrementar en acceso al sistema universitario, para verificar los argumentos descritos en el Cuadro 6 se presentan tres experimentos asumiendo falsamente que los instrumentos considerados incrementaron el acceso a estudios superiores en general, estudios superiores en universidades establecidas (más selectivas) y estudios terciarios o técnicos, los resultados de tales experimentos falsos se comparan con los resultados del experimento de interés. En general, tanto los efectos del acceso a estudios superiores como de los años de educación superior en los salarios se muestran positivos y significativos; mientras que, los efectos en la probabilidad de informalidad se muestran negativos y en algunos casos significativos.

No obstante, todos los experimentos falsos estimados en el contexto de la metodología IV-2SLS, no cumplirían algunas de las propiedades necesarias (verificables) para interpretar los efectos estimados como efectos causales (LATE o ACE); en particular, al considerar el efecto de los estudios universitarios se observa que los instrumentos serían instrumentos débiles dado que las pruebas-F de la primera etapa resultaron valores bajos, entre 2.41 y 9.35 siendo el valor crítico 11.51. Los efectos de la educación en los resultados laborales estimados en todos los experimentos falsos serían sesgados por la presencia de instrumentos débiles. Por su parte, los resultados de las pruebas de sobre-identificación de instrumentos, que evalúa la correlación entre el error predicho de la ecuación de salarios y los instrumentos (restricción de exclusión) sugieren es posible rechazar la hipótesis nula al $5 \%$ de significancia para todos los experimentos falsos, en tales casos es de esperar que $E\left[\varepsilon_{i}, d_{i}\right] \neq 0$. 
Cuadro 6: Efectos de la educación superior en los resultados laborales por tipo de estudios: estimaciones por IV

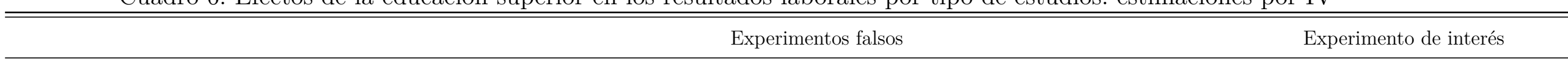

Estudios universitarios Estudios en universidades establecidas Estudios terciarios o técnicos Estudios en universidades entrantes

Panel A: Acceso a estudios superiores $\delta_{i v}^{l a t e}$

(Log)Salarios horarios

Probabilidad de informalidad

$0.226^{*}$

(0.127)

$-0.00875$

(0.132)

Primera etapa

F-Statistics

Prueba Hasen-Sargan (p-value)

8.35

0.08

$(2)$

$(3)$

$(4)$

Panel B: Años de estudios superiores $\delta_{i v}^{a c e}$

$(\mathrm{Log})$ Salarios horarios

Probabilidad de informalidad

$0.0577^{* *}$

$(0.0278)$

$-0.00796$

$(0.0295)$

$0.305^{* *}$

(0.136)

0.0152

(0.145)

$0.595 * * *$

$0.193)$

$-0.814^{* * *}$

$(0.298)$

$-0.520 * *$

(0.242)

2.41

12.46

0.02

0.40

0.06

Primera etapa

P-Statistics

Prueba Hasen-Sargan (p-value)

Observaciones

0.07

15,413

0.106

$0.135 * * *$

(0.0291)

$(0.0888)$

$-0.362^{* * *}$

(0.0450)

$-0.00478$

(0.132)

$-0.113^{* *}$

(0.0537)

Instrumentos: variables dummies por cada cohorte de nacimiento entre 1980 y 1990.

El grupo de control está constituido por individuos pertenecientes a las cohortes entre 1965 y 1979.

Probabilidad de informalidad: probabilidad que los trabajadores que no cuenten con ningún seguro de salud, ni cobertura de pensiones.

Todas las especificaciones incluyen como controles sexo, raza y efectos fijos a nivel de región de residencia.

La prueba $\mathrm{F}$ evalúa la hipótesis nula que los coeficientes asociados a las dummies de cohortes de nacimiento son conjuntamente iguales a cero.

Prueba Sargan-Hansen. Hipótesis nula: los instrumentos son válidos, no están correlacionados con el términos de error de la ecuación de salarios y que están correctamente excluidos.

Criterio de rechazo de hipótesis nula estadístico significativo, los resultados sugieren que no es posible rechazar hipótesis nula al $5 \%$ de significancia.

Clustered Standard errors in parentheses.

$* * * \mathrm{p}<0.01,{ }^{* *} \mathrm{p}<0.05, * \mathrm{p}<0.1$. 
La estrategia de identificación asume que las cohortes de individuos desde 1980 en adelante tuvieron mayor exposición al proceso de desregulación del sistema universitario afecto, ante ello cohortes anteriores no habrían sido expuestas a dicho proceso. Es posible verificar empíricamente que en cohortes anteriores a 1980 los instrumentos no tendrían efectos en los resultados laborales a través de la educación. En el panel A del Cuadro 7 se muestran los resultados de un experimento en el cual se asume falsamente que la desregulación del sistema universitario fue llevada a cabo en 1985 y afectó a la cohorte de individuos nacidos entre 1970 y 1979 (se considera aquí como grupo de control individuos nacidos entre 1965 y 1969). El estimado ACE aproximado por IV2SLS no resultó significativo estadísticamente, tanto en el efecto en salarios como en la probabilidad de informalidad.

Cuadro 7: Efectos de los años de educación superior en los resultados laborales por cohortes de años de nacimiento: estimaciones por IV

(Log)Salarios horarios Informalidad

(1)

\begin{tabular}{lcc} 
Panel A: Experimento falso & & \\
Cohorte de tratamiento:1970-1979 & & \\
Cohorte de control:1965-1969 & & -1.668 \\
$\delta_{i v}^{a c e}$ : Años de estudios universitarios (universidad entrante) & 0.319 & $(0.1726)$ \\
& $(0.234)$ & 4760 \\
Observations & 4179 & 0.01 \\
R-squared & 0.02 & \\
\hline Panel B: Experimento de interés & & \\
Cohorte de tratamiento:1980-1990 & & $-0.113^{* *}$ \\
Cohorte de control:1965-1979 & & $0.0537)$ \\
$\delta_{i v}^{a c e}:$ Años de estudios universitarios (universidad entrante) & $0.135^{* * *}$ & 9795 \\
& $(0.0449)$ & 0.058 \\
Observations & 8677 & \\
R-squared & 0.148 & \\
\hline
\end{tabular}

Instrumentos: variables dummies por cada cohorte de nacimiento.

El grupo de control está constituido por individuos pertenecientes a las cohortes entre 1965 y 1979.

Probabilidad de informalidad: probabilidad que los trabajadores que no cuenten con ningún seguro de salud, ni cobertura de pensiones.

Todas las especificaciones incluyen como controles sexo, raza y efectos fijos a nivel de región de residencia.

La prueba $\mathrm{F}$ evalúa la hipótesis nula que los coeficientes asociados a las dummies de cohortes de nacimiento son conjuntamente iguales a cero.

Clustered Standard errors in parentheses.

*** $\mathrm{p}<0.01, * * \mathrm{p}<0.05,{ }^{*} \mathrm{p}<0.1$. 


\section{Conclusiones}

En Perú en 1995 se implementó una reforma en el sistema universitario (desregulación), la cual permitió el ingreso de 84 nuevas universidades a nivel nacional (universidades entrantes al sistema) incrementando la oferta de universidades en $218 \%$ entre 1996 y 2017. Estas nuevas universidades tienen en general menores niveles de selectividad en el proceso de admisión de estudiantes y costos anuales más bajos que el promedio del sistema universitario peruano. Tales características generaron que una importante proporción de individuos accedan a estudios universitarios, los cuales en ausencia de la desregulación no habrían podido hacerlo y solamente contarían con estudios secundarios.

La desregulación del sistema universitario en 1995 generó que individuos nacidos luego del año 1980 sean el grupo de individuos más afectados por la reforma. En esta investigación se utiliza el año o cohorte de nacimiento como una característica exógena (instrumento) para implementar una estrategia de variables instrumentales que permita recuperar el efecto causal de la educación universitaria en algunos resultados laborales de interés, principalmente, salarios e informalidad laboral.

Si los supuestos de identificación son plausibles, se estima que por cada año de estudios universitarios en universidades entrantes los salarios se incrementan en 13.5 puntos porcentuales. Mientras que, el efecto acumulado del acceso a estudios universitarios es de 60 puntos porcentuales. No obstante, el efecto de la educación universitaria en los salarios es heterogéneo en función a características socioeconómicas, el efecto es cualitativamente mayor para mujeres (16 puntos porcentuales) y para individuos ubicados en el quintil más alto de ingresos familiares (16 puntos porcentuales).

La evidencia encontrada respecto del premio salarial de la educación universitaria (efecto de la educación superior en el capital humano) es relativamente similar al evidenciado por otros estudios a nivel internacional, los cuales también estimaron retornos salariales de la educación superior empleando variables instrumentales. En particular, [Maurin y McNally 2008], estiman un retorno de 14 puntos porcentuales por cada año de educación universitaria para el caso de una reforma universitaria en Francia y [Lemieux y Card 2001], quiénes estiman un retorno de 15 puntos porcentuales por año de educación universitaria para el caso de un programa educativo de larga escala en Canadá.

En contraste, para el caso peruano los estudios disponibles ha empleado básicamente ecuaciones de Mincer y, en general, estimado sus coeficientes de interés por OLS sin dar cuenta de los potenciales problemas de endogenidad en dicho estimador. Entre ellos:[Psacharopoulos 1994] estima retornos de 10 puntos porcentuales; mientras que, [Espinoza y Urzúa 2015], estiman que el retorno promedio de los estudios 
superiores en universidades entrantes sería de 24 puntos porcentuales. Finalmente [Yamada et al. 2015], quiénes emplean una aproximación por IV encuentran un premio salarial acumulado de la educación superior en universidades de menor calidad de 71 puntos porcentuales.

Este documento extiende la evidencia empírica previa para el caso peruano incluyendo efectos de la educación superior en las condiciones laborales de los trabajadores; en particular, en la informalidad laboral. Se encuentra que por cada año de estudios universitarios la probabilidad de informalidad en el empleo se reduce en 11.3 puntos porcentuales, reduciendo principalmente la probabilidad de no tener contrato de trabajo en 13 puntos porcentuales; sin embargo, no se encuentran efectos significativos en la probabilidad de no tener afiliación al pensiones jubilatorias, lo cual implicaría que existen dimensiones estructurales de informalidad en el mercado laboral peruano que no dependerían del nivel educativo de los trabajadores.

\section{Referencias}

[Angrist e Imbens 1995] Angrist, J. D. , and G. W. Imbens. Two-Stage Least Squares Estimation of Averge Causal Effects in Models with Variable Treatment Intensity, Journal of the American Statistical Association, 90:430, pp. 431-442.

[Angrist y Krueger 1991] Angrist, J. D. , and A. B. Krueger. Does compulsory school attendance affect schooling and earnings, The Quarterly Journal of Economics, Vol. 106, 1991, pp. 979-1014.

[Ashenfelter y Rouse 1998] Ashenfelter, O., and C. Rouse. Income, schooling and ability: evidence from a new sample of identical twins, The Quarterly Journal of Economics, Vol. 113, 1998, pp. 253-284.

[Ashenfelter y Zimmerman 1997] Ashenfelter, O., and D. Zimmerman. Estimates of the return to schooling from sibling data: fathers, sons and brothers, Review of Economics and Statistics, Vol. 79, 1997, pp. 1-9.

[Behrman et al. 1994] Behrman, J. R., R. Mark, R. Rosenzweig, and P. Taubman. Endowments and the allocation of schooling in the family and the marriage market: the twins experiment, Journal of Political Economy, Vol. 102, 1997, pp. 1131-1174.

[Borjas 2013] Borjas, G. Labor Economics, 6th ed. McGraw-Hill, NY, 2013.

[Card 1995] Card, D. Using geographic variation in college proximity to estimate de return to schooling, in L. N. Christofides, E. K. Grant, and R. Swidinsky Eds., As- 
pects of labor market behaviour: essays in honour of John Vanderkamp, University of Toronto Press, Toronto, 1995.

[Card 1999] Card, D. The Causal Effect of Education on Earnings Chapter 30 in O. Ashenfelter and D. Card Eds., Handbook of Labor Economics, Vol. 3 North-Holland, 1999.

[Dale y Krueger 2002] Dale, S. B., and A. B. Krueger. Estimating the Payoff to Attending a More Selective College: An Application of Selection on Observables and Unobservables, The Quarterly Journal of Economics, Vol. 117, No. 4, Nov. 2002, pp. 1491-1527.

[Duflo 2001] Duflo, E. Schooling and Labor Market Consecuences os School Construction in Indonesia: Evidence from an Unusual Policy Experiment. The American Economic Review, Vol. 91 No. 4, Sep. 2001, pp. 795-813.

[Espinoza y Urzúa 2015] Espinoza, R y S. Urzúa. The economics Returns to Higher Education: Funding, coverage and quality in Latin America Unpublished Working Paper, 2015

[Griliches 1977] Griliches, Z. Estimating the Returns to Schooling: Some Econometric Problems, Econometrica, Vol. 45, No. 1, Jan 1977, pp. 1-22.

[Holland 1986] Holland, P. Statistics and Causal Inference, Journal of the American Statistical Association, 81(1986), pp. 945-970.

[Imbens y Angrist 1994] Imbens, G. W., and J. Angrist. Identification and Estimation of Local Average Treatmet Effects, Econometrica, Vol. 62, No. 2, Mar. 1994, pp. 467-475.

[Lavado et al. 2014] Lavado, P., J.J. Martinez y G. Yamada. Una promesa incumplida? La calidad de la educación superior universitaria en el subempleo profesional en el Perú Serie de Documentos de Trabajo, DT. No. 2014-021, 2014, Banco Central de Reserva del Perú.

[Lavado et al. 2015] Lavado, P., J.J. Martinez y G. Yamada. Calidad de la educación superior y desigualdad en los retornos en el Perú Documentos de trabajo,No. 58, Diciembre 2015, Universidad del Pacífico.

[Lemieux y Card 2001] Lemieux, T., and D. Card. Education, Earnings, and the Canada G.I. Bill The Canadian Journal of Economcis, Vol. 34, No. 2, May 2001, pp. 313-344. 
[Loury y Garman 1995] Loury, L. D., and D. Garman. College Selectivity and Earnings, Journal of Labor Economics, Vol. 13, No. 2, Apr 1995, pp. 289-308.

[MacLeod et al. 2015] MacLeod, W. B., E. Riehl, J. E. Saavedra, and M. Urquiola. The Big Sort: College Reputation and Labor Market Outcomes, NBER Working Paper Series, WP 21230 June 2015, pp. 1-33.

[Maurin y McNally 2008] Maurin E., and S. McNally. Vive la Révolution! Long-Term Educational Returns of 1968 to the Angry Students, Journal of Labor Economics, Vol. 26, No. 1, Jan 2008, pp. 1-33.

[Mincer 1974] Mincer, J. Schooling, experience and earnings Columbia University Press, NY., 1974.

[Murphy y Welch 1990] Murphy, K. M., and F. Welch. Empirical Age-Earnings Profiles, Journal of Labor Economics, Vol. 8, No. 2, Apr 1990, pp. 202-229.

[Park 1996] Park, J. H. Measuring education over time: A comparison of old and new measures of education from the Current Population Survey, Economics Letters, 50 (1990), pp. 425-428.

[Psacharopoulos 1994] Psacharopoulos, G. Returns to Investment in Education: A Global Update, World Developmente, Vol. 22, No. 9 (1994), pp. 1325-1343.

[Rubin 1974] Rubin, D. Estimating Causal Effects of Treatments in Randomized and Nonrandomized Studies, Journal of Educational Psychology, 66(1974), pp. 688-701.

[Spence 1973] Spence, M. Job Market Signaling The Quarterly Journal of Economics, Vol. 87, No. 3 Aug. 1973, pp. 355-374.

[Staiger y Stock 1997] Staiger, D., and J. H. Stock. Instrumental variables regression with weak instruments Econometrica, Vol. 65, 1997, pp. 557-586.

[Stock y Yogo 2002] Stock J. H., and M. Yogo. Testing for Weak Instruments in Linear IV Regression NBER Technical Working Papers, Labor Studies, No. 284, 2002.

[Yamada et al. 2015] Yamada, G., P. LAvado y N. Oviedo. Valor de la información en Educación Superior y Efecto de la Calidad Universitaria en Remuneraciones en el Perú Documentos de trabajo,No. 57, Diciembre 2015, Universidad del Pacífico. 


\section{Apéndices}

Cuadro 8: Características básicas del sistema universitario peruano

\begin{tabular}{lcccc}
\hline \hline Características & $\begin{array}{c}\text { Establecidas } \\
\text { Públicas }\end{array}$ & $\begin{array}{c}\text { Establecidas } \\
\text { Privadas }\end{array}$ & $\begin{array}{c}\text { Entrantes } \\
\text { Públicas }\end{array}$ & $\begin{array}{c}\text { Entrantes } \\
\text { Privadas }\end{array}$ \\
\hline Año de creación & 1893 & 1975 & 2001 & 1997 \\
Índice de selectividad & $40 \%$ & $79 \%$ & $61 \%$ & $91 \%$ \\
Costo anual (en USD) & 73 & 2775 & 54 & 1865 \\
Alumnos matriculados & 326261 & 505027 & 19161 & 256975 \\
Participación & $29 \%$ & $46 \%$ & $2 \%$ & $23 \%$ \\
Ratio egresados/matriculados & 0.15 & 0.09 & 0.05 & 0.05 \\
Ratio titulados/matriculados & 0.11 & 0.06 & 0.02 & 0.03 \\
Ratio matriculados/docentes & 13 & 13 & 13 & 14 \\
\hline \hline
\end{tabular}

Establecidas Públicas y Privadas: universidades creadas antes de 1995.

Entrantes Públicas y Privadas: universidades creadas después de 1995.

Fuente: Superintendencia Nacional de Educación Superior (SUNEDU). 
Cuadro 9: Efectos de la educación en los resultados laborales por IV: primera etapa, 2SLS

Probabilidad de estudios universitarios Años de educación universitaria

(1)

(2)

\begin{tabular}{|c|c|c|}
\hline \multirow[t]{2}{*}{1980} & $0.042^{*}$ & $0.184^{*}$ \\
\hline & {$[1.9]$} & {$[1.85]$} \\
\hline \multirow[t]{2}{*}{1981} & $0.032 *$ & $0.151^{*}$ \\
\hline & {$[1.67]$} & {$[1.71]$} \\
\hline \multirow[t]{2}{*}{1982} & $0.048^{* * *}$ & $0.219 * * *$ \\
\hline & {$[2.74]$} & {$[2.76]$} \\
\hline \multirow[t]{2}{*}{1983} & $0.035^{* *}$ & $0.164^{* *}$ \\
\hline & {$[2.13]$} & {$[2.19]$} \\
\hline \multirow[t]{2}{*}{1984} & $0.061 * * *$ & $0.288^{* * *}$ \\
\hline & {$[2.69]$} & {$[2.82]$} \\
\hline \multirow[t]{2}{*}{1985} & $0.081^{* * *}$ & $0.387^{* * *}$ \\
\hline & {$[3.41]$} & {$[3.45]$} \\
\hline \multirow[t]{2}{*}{1986} & $0.084^{* * *}$ & $0.388^{* * *}$ \\
\hline & {$[3.61]$} & {$[3.63]$} \\
\hline \multirow[t]{2}{*}{1987} & $0.125^{* * *}$ & $0.556^{* * *}$ \\
\hline & {$[4.44]$} & {$[4.37]$} \\
\hline \multirow[t]{2}{*}{1988} & $0.132^{* * *}$ & $0.570^{* * *}$ \\
\hline & {$[4.43]$} & {$[4.45]$} \\
\hline \multirow[t]{2}{*}{1989} & $0.153^{* * *}$ & $0.696^{* * *}$ \\
\hline & {$[5.10]$} & {$[5.31]$} \\
\hline \multirow[t]{2}{*}{1990} & $0.186^{* * *}$ & $0.842^{* * *}$ \\
\hline & {$[6.00]$} & {$[6.09]$} \\
\hline \multirow[t]{2}{*}{ Cohort-trend } & 0.003 & 0.014 \\
\hline & {$[0.30]$} & {$[0.34]$} \\
\hline \multirow[t]{2}{*}{ Edad } & 0.003 & 0.015 \\
\hline & {$[0.32]$} & {$[0.37]$} \\
\hline \multirow[t]{2}{*}{ Sexo (1: varón) } & $-0.036^{* * *}$ & $-0.170^{* * *}$ \\
\hline & {$[-2.79]$} & {$[-2.84]$} \\
\hline \multirow[t]{2}{*}{ Raza (1: indigena) } & $-0.076^{* * *}$ & $-0.342^{* * *}$ \\
\hline & {$[-8.01]$} & {$[-7.90]$} \\
\hline F-statistics & 12.46 & 11.83 \\
\hline Observations & 8,677 & 8,678 \\
\hline
\end{tabular}

Todas las especificaciones incluyen como controles sexo, raza y efectos fijos a nivel de región de residencia. El grupo de control está constituido por individuos pertenecientes a las cohortes entre 1965 y 1979.

La prueba $\mathrm{F}$ evalúa la hipótesis nula que los coeficientes asociados a las dummies de cohortes de nacimiento son conjuntamente iguales a cero.

Robust t-statistics in brakets.

$* * * \mathrm{p}<0.01,{ }^{* *} \mathrm{p}<0.05,{ }^{*} \mathrm{p}<0.1$. 
Cuadro 10: Pruebas de especificación IV-2SLS

Probabilidad de estudios universitarios Años de educación universitaria

(1)

$(2)$

\begin{tabular}{lcc} 
& $(1)$ & $(2)$ \\
Prueba de Hausman & 1.29 & 1.45 \\
p-value & $(0.256)$ & $(0.228)$ \\
\hline Prueba de sobreidentificación & & 1.01 \\
Prueba-F & 1.02 & $(0.434)$ \\
p-value & $(0.426)$ & 16.91 \\
Prueba Hasen-Sargan (Chi-2, errores robustos) & 17.52 & $(0.074)$ \\
p-value & $(0.0636)$ & \\
\hline \hline
\end{tabular}

Prueba de Hausman. Hipótesis nula: estimador OLS es consistente y eficiente; hipótesis alterna: estimador OLS es inconsistente, por construcción estimador IV-2SLS siempre es consistente. Criterio de no aceptación de hipótesis nula estadístico no significativo.

Prueba-F, significancia conjunta de regresar los errores predichos de la ecuación de salarios sobre los instrumentos, hipótesis nula: los coeficientes asociados a los instrumentos son conjuntamente distintos de cero, los resultados sugieren que no es posible rechazar hipótesis nula.

Prueba Sargan-Hansen. Hipótesis nula: los instrumentos son válidos, no están correlacionados con el términos de error de la ecuación de salarios y que están correctamente excluidos. Criterio de rechazo de hipótesis nula estadístico significativo, los resultados sugieren que no es posible rechazar hipótesis nula al $5 \%$ de significancia. 
Figura 6: Escolaridad y año de nacimiento por sexo

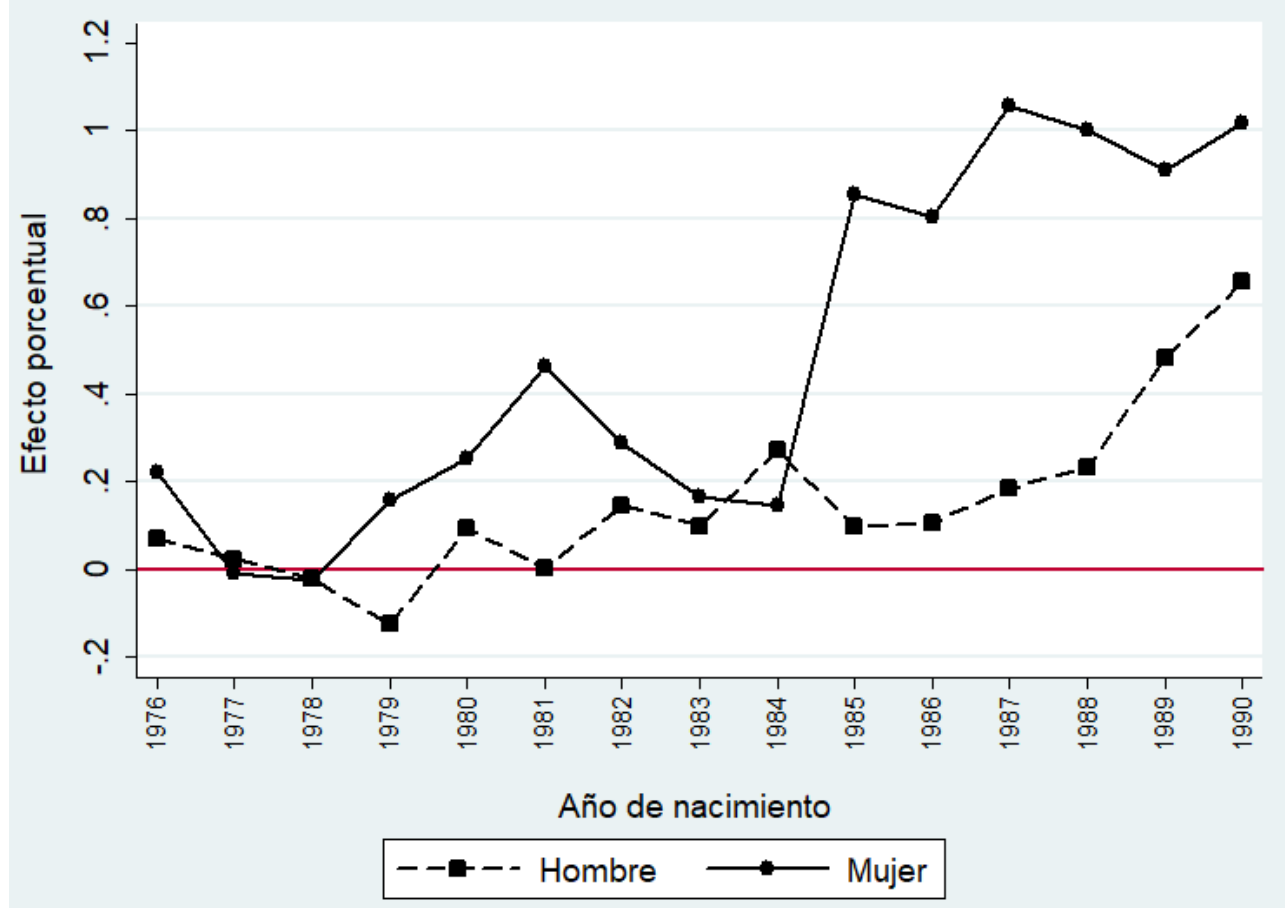

Estimaciones correspondientes al modelo de forma reducida.

Escolaridad: años de educación.

Fuente: Elaboración Propia.

Figura 7: Escolaridad y año de nacimiento por raza

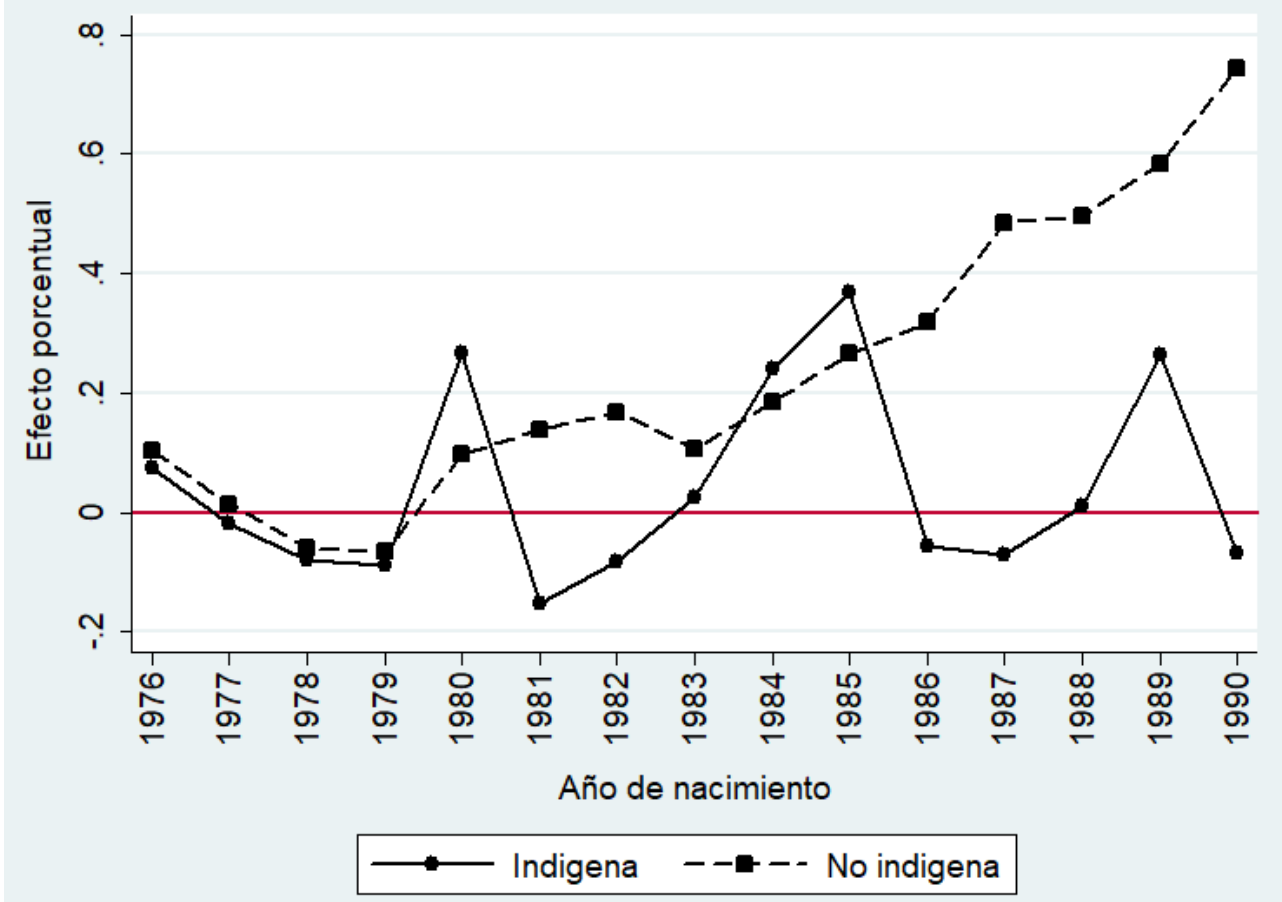

Estimaciones correspondientes al modelo de forma reducida.

Escolaridad: años de educación.

Fuente: Elaboración Propia. 
Figura 8: Escolaridad y año de nacimiento por quintiles del ingreso familiar per cápita

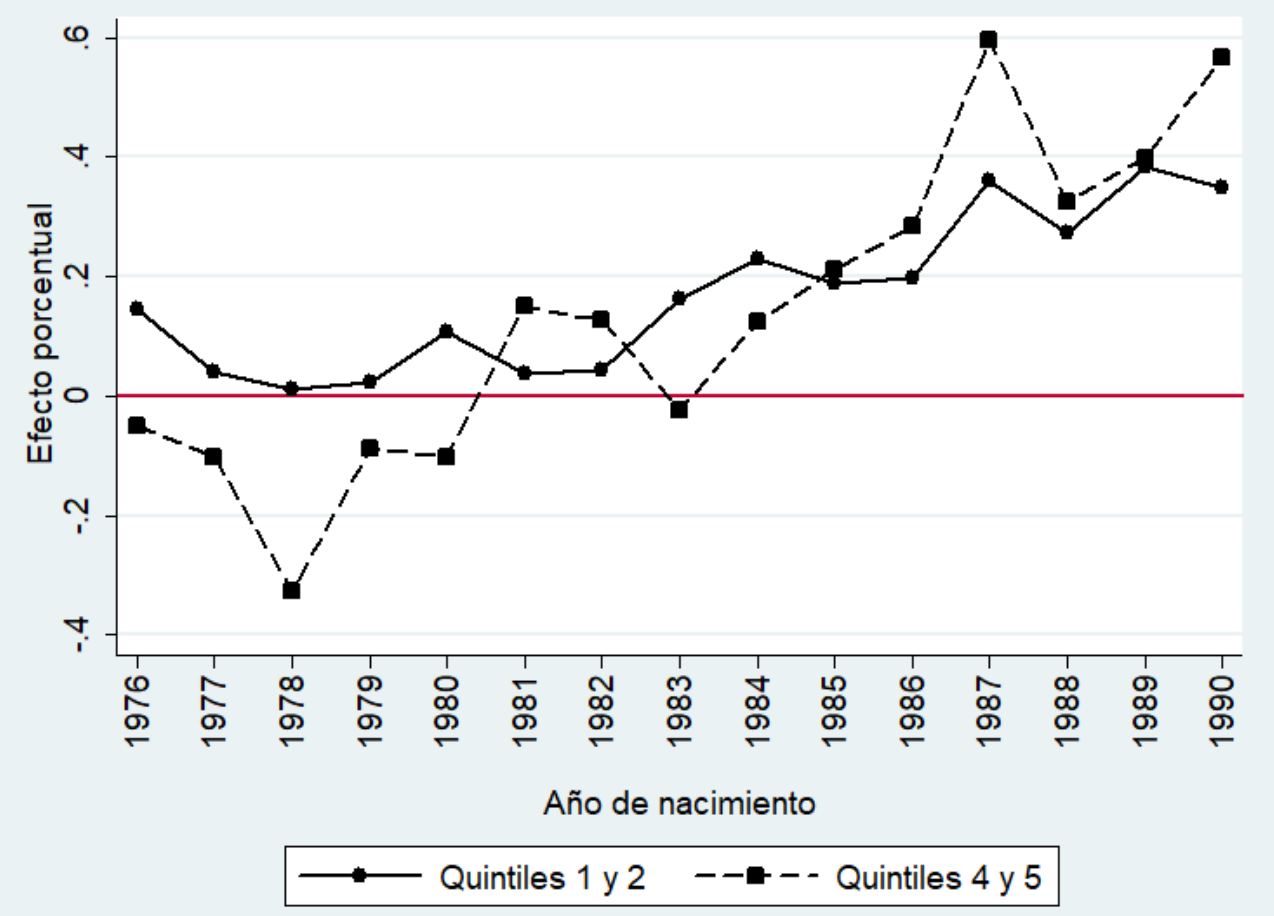

Estimaciones correspondientes al modelo de forma reducida. Escolaridad: años de educación.

Fuente: Elaboración Propia. 